\title{
VALOR TAXONÓMICO DEL POLEN EN SCROPHULARIACEAE SENSU LATO
}

\author{
MARÍA DE LAS MERCEDES SOSA ${ }^{1,3}$ y CRISTINA R. SALGADO 2,3
}

\begin{abstract}
Resumen: Este trabajo es el resultado del estudio palinomorfológico de 32 especies pertenecientes a 19 géneros de Scrophulariaceae sensu lato. Además se realizó un registro exhaustivo de la información bibliográfica existente sobre la morfología polínica con el objetivo de analizar las relaciones entre las variables palinológicas y la clasificación sistemática actual. Los granos de polen analizados son: mónades, radiosimétricos e isopolares, de tamaño variable desde pequeños $(11 \mu \mathrm{m})$ hasta medianos $(51 \mu \mathrm{m})$, predominantemente esferoidales $(P / E=1)$, a veces suboblatos $(P / E=0,76)$, oblato-esferoidales $(P / E=0,95)$, prolatos $(P / E=1,63)$ y perprolatos $(P / E=2,63)$. Inaperturados, 3-(4-5) colpados, 3- $(4-5)$ colporados, espiraperturados, 2-8 sincolpados, 3-diploporados y pantoporados. La exina puede ser semitectada (retipilada, reticulada o estriada perforada) y tectada (psilada, foveolada, perforada, escábrida, rugulada, verrugosa, granulada, estriada o equinulada). Se realizó un análisis estadístico en base a una matriz de datos, compuesta por los caracteres palinológicos de 32 especies estudiadas sumado a la recopilación bibliográfica de 140 géneros y 218 especies pertenecientes a Scrophulariaceae sensu lato. El análisis de agrupamiento (UPGMA), confirmó que las diferencias palinomorfológicas apoyan la clasificación sistemática actual.
\end{abstract}

Palabras clave: Morfología del polen, sistemática, euripolínico, Scrophulariaceae.

Summary: Taxonomic value of pollen in Scrophulariaceae sensu lato. This paper is result of a study of pollen morphology of 32 species belonging to 19 genera of Scrophulariaceae sensu lato. A comprehensive record of available information on the pollen morphology in this group in order to analyze the relationships between palynological variables and the current systematic classification was also conducted. Pollen grains are monads, radially symetric and isopolar, the sizes vary from small $(11 \mu \mathrm{m})$ to medium $(51 \mu \mathrm{m})$, predominately spheroidal $(P / E=1)$, sometimes suboblates $(P / E=0.76)$, oblate-spheroidal $(P / E=0.95)$, prolates $(P / E=1.63)$ and perprolates $(P / E=2.63)$. They are inaperturates, 3- $(4-5)$ colpates, 3- $(4-5)$ colporates, spiraperturates 2-8 syncolpates, 3-colpo-diploporates and pantoporates grains. The exine may be semitectate (retipilate, reticulate or striato-perforate) and tectate (psilate, foveolate, perforate, scabrate, rugulate, verrucate, granulose, striate, echinate). Statistical analysis was performed based on the data matrix of the palynological characters of the 32 species studies in addition to bibliographic references of 140 genera and 218 species belonging to Scrophulariaceae sensu lato. The cluster analysis (UPGMA), confirmed that palynomorphological differences support the current systematic classification.

Key words: Pollen morphology, systematic, eurypalynous, Scrophulariaceae.

\section{INTRODUCCIÓN}

La familia Scrophulariaceae sensu lato ha sido conocida durante mucho tiempo como una gran

${ }^{1}$ Facultad de Ciencias Exactas y Naturales y Agrimensura, UNNE. Av. Libertad 5460. 3400. Corrientes, Argentina. E-mail: mdlmsvg@yahoo.com.ar

2 Facultad de Ciencias Agrarias, UNNE. Sgto. Cabral 2131. 3400. Corrientes, Argentina. E-mail: polenenmiel@agr. unne.edu.ar

${ }^{3}$ Instituto de Botánica del Nordeste, UNNE - CONICET. Sgto Cabral 2131. 3400. Corrientes, Argentina. familia dentro del orden Lamiales (Bentham, 1846, 1876; Wettstein, 1897), muy heterogénea por lo que no constituye un grupo taxonómico natural (Thieret, 1967). Desde entonces hasta la fecha, ha sufrido modificaciones en su delimitación, con la incorporación de familias como Selaginaceae (Bentham, 1846) o el reconocimiento de familias derivadas como Lentibulariaceae, Plantaginaceae y Orobanchaceae (Wettstein, 1897). En ese aspecto, Cronquist (1981) lo removió del orden Lamiales y lo elevó a nivel de orden Scrophulariales, al igual que Plantaginaceae, que también, de acuerdo a su criterio, pertenecería al orden Plantaginales. Es decir, que a lo largo de sucesivas revisiones, su 
circunscripción ha sido constantemente discutida, pero sobre todo en los últimos 20 años, donde con la incorporación de la filogenia molecular se ha demostrado que se trata de una familia polifilética (Olmstead \& Reeves, 1995; Olmstead et al., 2001; Albach et al., 2005; Oxelman et al., 2005; Rahmanzadeh et al., 2005; Bennett \& Mathews, 2006; Tank et al., 2006). Estos estudios filogenéticos moleculares revelaron que la familia no presenta un conjunto de características derivadas de forma única (ausencia de sinapomorfías), lo que no permite reconocer fácilmente a sus integrantes del resto de las familias estrechamente relacionadas (Tank et al., 2006). La polifilia de la familia fue demostrada por Olmstead \& Reeves (1995), con unos pocos géneros, avalando dos clados: "scroph I" que incluía a Scrophulariaceae sensu stricto y "scroph II" con géneros de climas templados como Anthirrinum L., Digitalis L. y Veronica L.; y los géneros considerados en ese momento pertenecientes a familias monogenéricas como Plantago L., Hippuris L. y Callitriche L. Esto fue confirmado en sucesivos estudios filogenéticos, lo que llevó a la reestructuración de la familia, de tal manera que varios géneros o tribus que pertenecían a Scrophulariaceae; fueron trasladados a diferentes familias ya existentes y otras nuevas. En ese contexto, de acuerdo al sistema de clasificación actual (APG III, 2009 y Stevens, 2001, onwards) se conocen las siguientes familias como derivadas de Scrophulariaceae sensu lato, ellas son Calceolariaceae, Linderniaceae, Mazaceae, Orobanchaceae, Paulowniaceae, Plantaginaceae, Phrymaceae, Scrophulariaceae y Stilbaceae; también se incorporaron Buddlejaceae, Globulariaceaae y Myoporaceae dentro de estas familias.

La morfología polínica como carácter taxonómico para muchos grupos de plantas tiene relevancia en los sistemas modernos de clasificación (APG III) donde su uso alcanza destacada importancia. Por otra parte, el estudio morfológico del polen puede contribuir al establecimiento de relaciones filogenéticas entre los taxones. En ese aspecto en Scrophulariaceae sensu lato, autores como Varghese (1968), Karim \& El-Oqlah (1989), Inceoglu (1982), Hong (1984), Argue (1980, 1981, 1984, 1985, 1986, 1990, 1993), Bigazzi \& Tardelli (1990), Correa et al. (1995), Fernández et al. (1997), Santos \& Melhem (1999, 2000), Wang et al. (2003,
2009), Saeidi-Mehrvarz \& Zarrei (2006); Kheiri et al. (2006) destacaron el valor taxonómico del polen que permitió en algunos casos separar tribus, géneros y especies.

En este trabajo se describe la morfología polínica de 32 especies pertenecientes a 19 géneros de la familia Scrophulariaceae sensu lato. Los resultados obtenidos, junto a los provenientes de la recopilación bibliográfica realizada, fueron incorporados a una base de datos con el objetivo de definir el valor de los caracteres palinológicos para la delimitación de los grupos taxonómicos actuales.

\section{Materiales y Métodos}

Se seleccionaron 47 especímenes pertenecientes a 32 especies de Scrophulariaceae sensu lato provenientes del Herbario CTES (Tabla 1). Los botones florales a punto de antesis fueron procesados según la técnica de acetólisis (Erdtman, 1960). Los preparados permanentes fueron incluidos a la Palinoteca CTES y analizados con microscopio óptico (MO). Se realizaron mediciones del diámetro polar, diámetro ecuatorial y se calculó la forma de 30 granos de polen por espécimen. La escultura de la exina fue analizada con microscopio electrónico de barrido (MEB) (JEOL $5800 \mathrm{LV}$ ), para ello, los granos fueron montados sobre láminas de aluminio, secados a temperatura ambiente, cubiertos con oro y adheridos con cinta bifaz al stub. La terminología utilizada para realizar las descripciones es la sugerida por Sáenz Laín (2004) y Punt et al. (2007). Los datos palinológicos de las especies analizadas sumados a los provenientes de una exhaustiva recopilación bibliográfica se incorporaron a una base de datos conformada por 140 géneros y 218 especies que fueron ubicadas en las actuales familias de Calceolariaceae, Linderniaceae, Mazaceae, Orobanchaceae, Phrymaceae, Plantaginaceae y Scrophulariaceae (Tabla 2) para confirmar si hay una correlación entre la morfología polínica y la clasificación actual.

Con el fin de evaluar la similitud de las características palinológicas de las especies se realizó un análisis de agrupamiento. La matriz de datos quedó constituida por 240 unidades taxonómicas operacionales (OTUs) por 7 variables: diámetro polar $(\mathrm{P})$, diámetro ecuatorial $(\mathrm{E})$, forma del grano $(\mathrm{P} / \mathrm{E})$, estructura (Struc.) y escultura 
Tabla 1. Material estudiado con sus respectivas localidades, colecccionistas y número de PAL-CTES.

\begin{tabular}{|c|c|c|c|c|}
\hline Familia & Género & Especie & Localidad, Colector y Número & $\begin{array}{l}\text { Número de } \\
\text { PAL-CTES }\end{array}$ \\
\hline \multirow[t]{2}{*}{ Calceolariaceae } & Calceolaria & C. elatior Griseb. & $\begin{array}{l}\text { ARGENTINA. Prov. Jujuy, Dpto. Ledesma, } \\
\text { Parque Nacional Calilegua. 2-XI-1998, } \\
\text { Ahumada \& Agüero } 8518 \text { (CTES). }\end{array}$ & 4305 \\
\hline & Jovellana & $\begin{array}{l}\text { J. violacea (Cav.) } \\
\text { G. Don. }\end{array}$ & $\begin{array}{l}\text { CHILE. Concepción, Quebrada Honda, 1-XII- } \\
\text { 1976, Marticorena et al. } 1135 \text { (CTES). }\end{array}$ & 4306 \\
\hline \multirow[t]{3}{*}{ Linderniaceae } & Lindernia & L. dubia (L) Pennell & $\begin{array}{l}\text { ARGENTINA. Prov. Corrientes: Dpto. San } \\
\text { Cosme, Paso de la Patria, 23-XI-1981, Cristóbal } \\
\text { \& Schinini } 1850 \text { (CTES). }\end{array}$ & 2854 \\
\hline & & & $\begin{array}{l}\text { ARGENTINA. Prov. Chaco: Dpto. Bermejo, Isla } \\
\text { Brasilera, 4-IX-1965, Schulz } 15141 \text { (CTES). }\end{array}$ & 2856 \\
\hline & Torenia & $\begin{array}{l}\text { T. thouarsii (Cham. } \\
\text { \& Schltdl.) Kuntze }\end{array}$ & $\begin{array}{l}\text { BRASIL. Bahía, Nova Viscosa,8-XI-1986, } \\
\text { Hatschbach \& Silva } 50739 \text { (CTES). }\end{array}$ & 4307 \\
\hline \multirow[t]{4}{*}{ Phrymaceae } & Mimulus & M. bridgesii Clos. & $\begin{array}{l}\text { CHILE. VII Región, Camino de Angol a Los } \\
\text { Alpes, 29-XI-2008, Morrone et al. } 6016 \text { (CTES). }\end{array}$ & 4315 \\
\hline & & M. cupreus Dombrain & $\begin{array}{l}\text { ARGENTINA. Prov. Neuquén: Dpto. Chapelco, } \\
\text { 4-II-1977, Crespo \& Giangualani s/n (CTES). }\end{array}$ & 4316 \\
\hline & & M. glabratus Kunth. & $\begin{array}{l}\text { ARGENTINA. Prov.Tucumán: Dpto. Tafí; } 30- \\
\text { VIII-1971, Krapovickas \& Cristóbal } 20520 \\
\text { (CTES). }\end{array}$ & 4317 \\
\hline & & M. luteus L. & $\begin{array}{l}\text { ARGENTINA. Prov. Mendoza: Dpto. Malargüe; } \\
\text { 25-II-2003, Prina et al. } 1954 \text { (CTES). }\end{array}$ & 4318 \\
\hline \multirow[t]{10}{*}{ Orobanchaceae } & Agalinis & $\begin{array}{l}\text { A. communis (Cham. } \\
\text { \& Schltdl) D'Arcy }\end{array}$ & $\begin{array}{l}\text { ARGENTINA. Prov. Corrientes: Dpto. Santo } \\
\text { Tomé, Estancia Garruchos, 11-II-1972, } \\
\text { Krapovickas et al. } 21610 \text { (CTES). }\end{array}$ & 2656 \\
\hline & & & $\begin{array}{l}\text { ARGENTINA. Prov. Misiones: Dpto. Capital, } \\
\text { Bañado del arroyo Zaimán, 21-IX-1994, Guillen } \\
\text { et al. } 359 \text { (CTES). }\end{array}$ & 6698 \\
\hline & & $\begin{array}{l}\text { A. genistifolia (Cham. } \\
\text { \& Schltdl.) D'Arcy }\end{array}$ & $\begin{array}{l}\text { ARGENTINA. Prov. Chaco: Dpto. Tapenagá, } \\
\text { Enrique Urien, 24-II-1951, Schulz } 8233 \text { (CTES). }\end{array}$ & 6702 \\
\hline & & & $\begin{array}{l}\text { ARGENTINA. Prov. Misiones: Dpto. Candelaria, } \\
\text { ruta 12, } 18 \text { km NE de Candelaria, 21-III-1997, } \\
\text { Tressens et al. } 5727 \text { (CTES). }\end{array}$ & 6701 \\
\hline & Buchnera & $\begin{array}{l}\text { B. integrifolia } \\
\text { Larrañaga }\end{array}$ & $\begin{array}{l}\text { ARGENTINA. Prov. Corrientes: Dpto. } \\
\text { Mburucuyá, Parque Nacional Mburucuyá, 06- } \\
\text { I-2004, Sosa } 126 \text { (CTES). }\end{array}$ & 2651 \\
\hline & & & $\begin{array}{l}\text { ARGENTINA. Prov. Misiones: Dpto. San Javier, } \\
\text { ruta 4, camino a San Javier, 13-III-1969, } \\
\text { Krapovickas et al. } 15194 \text { (CTES). }\end{array}$ & 2650 \\
\hline & & B. longifolia Kunth. & $\begin{array}{l}\text { ARGENTINA. Prov. Corrientes: Dpto. Santo } \\
\text { Tomé, ruta 38, 1-XII-1981, Tressens et al. } 1388 \\
\text { (CTES). }\end{array}$ & 2653 \\
\hline & & & $\begin{array}{l}\text { ARGENTINA. Prov. Misiones: Dpto. San } \\
\text { Ignacio, Santo Pipo, 23-XI-2003, Cocucci et al. } \\
3163 \text { (CTES). }\end{array}$ & 2652 \\
\hline & & B. ternifolia Kunth. & $\begin{array}{l}\text { ARGENTINA. Prov. Corrientes, Dpto. Santo } \\
\text { Tomé, ruta prov. } 40,07-X I I-1997, \text { Múlgura de } \\
\text { Romero et al. } 1562 \text { (CTES). }\end{array}$ & 2654 \\
\hline & & & $\begin{array}{l}\text { ARGENTINA. Prov. Misiones: Dpto. San } \\
\text { Ignacio, San Ignacio, casa Quiroga, 13-II-1976, } \\
\text { Krapovickas \& Cristóbal } 28695 \text { (CTES). }\end{array}$ & 2655 \\
\hline
\end{tabular}




\begin{tabular}{|c|c|c|c|c|}
\hline Familia & Género & Especie & Localidad, Colector y Número & $\begin{array}{l}\text { Número de } \\
\text { PAL-CTES }\end{array}$ \\
\hline & Castilleja & $\begin{array}{l}\text { C. lithospermoides } \\
\text { Kunth. }\end{array}$ & $\begin{array}{l}\text { ARGENTINA. Prov. Corrientes, Dpto. Santo } \\
\text { Tomé, ruta } 40 \text { y Arroyo Chimiray, 12-XI-1974, } \\
\text { Schinini \& Carnevali } 10673 \text { (CTES). }\end{array}$ & 2642 \\
\hline & & & $\begin{array}{l}\text { ARGENTINA. Prov. Misiones: Dpto. Capital, } \\
\text { ruta prov. N }{ }^{\circ} 1,12 \mathrm{~km} \text { S de Posadas, 16-XI- } \\
\text { 1974, Schinini \& Carnevali } 10704 \text { (CTES). }\end{array}$ & 2643 \\
\hline & Dasistoma & D. macrophyllum Raf. & $\begin{array}{l}\text { ESTADOS UNIDOS: Oklahoma, Murray, 11-VII- } \\
\text { 1970, Correll \& Correll } 39027 \text { (CTES). }\end{array}$ & 4308 \\
\hline & Escobedia & $\begin{array}{l}\text { E. grandiflora } \\
\text { (L. f.) Kuntze }\end{array}$ & $\begin{array}{l}\text { ARGENTINA. Prov. Corrientes: Dpto. San } \\
\text { Miguel, Estancia Curuzú Laurel, 31-III-1974, } \\
\text { Krapovickas et al. } 24729 \text { (CTES). }\end{array}$ & 2641 \\
\hline & & & $\begin{array}{l}\text { ARGENTINA. Dpto. Santo Tomé, } \\
\text { Establecimiento Las Marías, 1-XII-1970, } \\
\text { Krapovickas et al. } 16793 \text { (CTES). }\end{array}$ & 2640 \\
\hline & Melasma & $\begin{array}{l}\text { M. melampyroides } \\
\text { (Rich.) Pennell }\end{array}$ & $\begin{array}{l}\text { ARGENTINA. Prov. Corrientes, Dpto. Santo } \\
\text { Tomé, Estancia Garruchos, 10-II-1972, } \\
\text { Krapovickas et al. } 21454 \text { (CTES). }\end{array}$ & 6703 \\
\hline & & & $\begin{array}{l}\text { ARGENTINA. Prov. Misiones: Dpto. Capital, } \\
\text { Bañado Arroyo Zaimán, 16-XI-1994, Lirussi et } \\
\text { al. } 587 \text { (CTES). }\end{array}$ & 6704 \\
\hline & & & $\begin{array}{l}\text { ARGENTINA. Prov. Misiones. Dpto. San Pedro, } \\
\text { ruta Nac. } 14 \text {, Palmera Boca, 10-III-2008, Keller } \\
\& \text { Franco } 5110 \text { (CTES). }\end{array}$ & 2645 \\
\hline & & $\begin{array}{l}\text { M. rhinanthoides } \\
\text { (Cham.) Benth. }\end{array}$ & $\begin{array}{l}\text { ARGENTINA. Prov. Corrientes: Dpto. Santo } \\
\text { Tomé, Estancia Timbó, 28-XI-1983, Schinini et } \\
\text { al. } 23607 \text { (CTES) }\end{array}$ & 2646 \\
\hline & & & $\begin{array}{l}\text { ARGENTINA. Prov. Misiones: Dpto. Iguazú, } \\
\text { Bañado la Alpargata, 1-IV-2002, Keller et al. } \\
1739 \text { (CTES). }\end{array}$ & 2647 \\
\hline & & $\begin{array}{l}\text { M. stricta (Benth.) } \\
\text { Hassl. }\end{array}$ & $\begin{array}{l}\text { ARGENTINA. Prov. Corrientes: Dep. Ituzaingó, } \\
\text { Estancia Tavé Retá, 26-IV-1974, Pedersen } \\
9156 \text { (CTES). }\end{array}$ & 2648 \\
\hline & & & $\begin{array}{l}\text { PARAGUAY. Amambay, Camino entre ruta } 5 \text { y } \\
\text { Bella Vista, 15-V-1974, Schinini } 8949 \text { (CTES). }\end{array}$ & 2649 \\
\hline & Orobanche & O. alba Stephan. & $\begin{array}{l}\text { SUIZA. Zurich; 6-VIII-1951, Kummer s/n } \\
\text { (CTES). }\end{array}$ & 4310 \\
\hline & & O. lutea Baumg. & $\begin{array}{l}\text { AUSTRIA. Viena; 29-V-1921, Sulger Büel s/n } \\
\text { (CTES). }\end{array}$ & 4311 \\
\hline & & O. minor Sm. & $\begin{array}{l}\text { AUSTRIA. Karnten, Hermagor, Kötschach; 28- } \\
\text { VI-1963, Pedersen } 6797 \text { (CTES). }\end{array}$ & 4312 \\
\hline & & O. racemosa Vell. & $\begin{array}{l}\text { SUIZA. Zurich; 28-VIII-1942, Tessin } 33992 \\
\text { (CTES). }\end{array}$ & 4313 \\
\hline & & O. reticulata Wallr. & $\begin{array}{l}\text { SUIZA. St. Gallen; 6-X-1940, Sulger Büel s/n } \\
\text { (CTES). }\end{array}$ & 4314 \\
\hline & Seymeria & S. deflexa Eastw. & $\begin{array}{l}\text { MÉXICO. Estado Nuevo León, Sierra Madre; } \\
\text { 19-XI-1907, Pringle } 10398 \text { (CTES). }\end{array}$ & 4309 \\
\hline Plantaginaceae & Mecardonia & $\begin{array}{l}\text { M. grandiflora } \\
\text { (Benth.) Pennell }\end{array}$ & $\begin{array}{l}\text { ARGENTINA. Prov. Misiones: Dpto. San Pedro, } \\
\text { Saltos del Moconá, 7-V-1999, Deginani et al. } \\
1112 \text { (CTES). }\end{array}$ & 2950 \\
\hline & & & $\begin{array}{l}\text { ARGENTINA. Dpto. Iguazú, P.N. Iguazú, 6-X- } \\
\text { 1994, Herrera } 21 \text { (CTES). }\end{array}$ & 2951 \\
\hline & Plantago & P. lanceolata L. & $\begin{array}{l}\text { ARGENTINA. Prov. Salta. Dpto. Chicoana. } \\
\text { Zuloaga et al. } 9392 \text { (CTES). }\end{array}$ & 4319 \\
\hline
\end{tabular}


M. M. Sosa y C. R. Salgado - Palinotaxonomía de Scrophulariaceae sensu lato

\begin{tabular}{|c|c|c|c|c|}
\hline Familia & Género & Especie & Localidad, Colector y Número & $\begin{array}{l}\text { Número de } \\
\text { PAL-CTES }\end{array}$ \\
\hline & Stemodia & S. Iobelioides Lehm. & $\begin{array}{l}\text { ARGENTINA. Prov. Corrientes: Dpto. Monte } \\
\text { Caseros; 6-V-2005, Sosa \& Schinini } 210 \\
\text { (CTES). }\end{array}$ & 2540 \\
\hline \multirow[t]{6}{*}{ Scrophulariaceae } & Buddleja & $\begin{array}{l}\text { B. grandiflora } \\
\text { Cham. \& Schltdl. }\end{array}$ & $\begin{array}{l}\text { ARGENTINA. Prov. Chaco: Dpto. Presidente } \\
\text { Roque Sáenz Peña, XI-1939, Di Fonzo No } 7 \\
\text { (CTES). }\end{array}$ & 4321 \\
\hline & & B. tubiflora Benth. & $\begin{array}{l}\text { ARGENTINA. Prov. Formosa: Dpto. El } \\
\text { Colorado, 8-VIII-1963, Schulz 422(CTES). }\end{array}$ & 4322 \\
\hline & Capraria & C. biflora L. & $\begin{array}{l}\text { ARGENTINA. Prov. Formosa: Dpto. Bartolomé } \\
\text { de las Casas, IX-1971, Insfran } 915 \text { (CTES). }\end{array}$ & 2657 \\
\hline & & & $\begin{array}{l}\text { ARGENTINA. Prov. Salta: Dpto. Rivadavia, } \\
\text { Santa Victoria Este, 21-XII-2002, Scarpa } 470 \\
\text { (SI, CTES). }\end{array}$ & 2659 \\
\hline & Verbascum & V. virgatum Stokes. & $\begin{array}{l}\text { ARGENTINA. Prov. Corrientes: Dpto. Paso de } \\
\text { los Libres, puente Miriñay, 5-XI-1976, Pedersen } \\
11363 \text { (CTES) }\end{array}$ & 2660 \\
\hline & & & $\begin{array}{l}\text { ARGENTINA. Prov. Chaco: Dpto. Fontana, } \\
\text { Urien, X-1932, Schulz } 1007 \text { (CTES). }\end{array}$ & 2661 \\
\hline
\end{tabular}

(Scult.) de la exina, tipo y número de aperturas (Tabla 3).

La matriz de distancia se construyó a partir de la matriz de datos y del coeficiente de distancia Euclídea. Para agrupar las OTUs se utilizó el método de pares no ponderados usando las medias aritméticas (UPGMA), además se calculó el coeficiente de correlación cofenético como una medida de la distorsión introducida durante el agrupamiento. Los análisis se llevaron a cabo con el software InfoStat, versión 2013 (Di Rienzo et al., 2013).

\section{Resultados}

\section{Análisis polínico}

A partir del estudio palinomorfológico de las 32 especies seleccionadas se determinaron 17 tipos polínicos (Tabla 4). Las variables con mayor diversidad fueron las aperturas -tipo y número- y la estructura y escultura de la exina.

Los granos de polen son liberados en forma de mónade. La totalidad de los granos descriptos son radiosimétricos e isopolares. El tamaño de los granos de polen varía desde pequeños (11 $\mu \mathrm{m})$ a medianos $(51 \mu \mathrm{m})$. Generalmente son esferoidales $(\mathrm{P} / \mathrm{E}=1)$ pero también se conocen granos suboblatos $(\mathrm{P} / \mathrm{E}=0,76)$, oblato-esferoidales $(\mathrm{P} / \mathrm{E}=0,95)$ y prolatos $(\mathrm{P} / \mathrm{E}=1,5)$. En cuanto a las aperturas se describieron granos inaperturados, 3-(4) colpados, 3-(4-5) colporados, espiraperturados, 3-diploporados y pantoporados (7-14). La exina puede ser semitectada (retipilada, reticulada o retículo-rugulada) o tectada (psilada, foveolada, perforada, escábrida-verrugosa, rugulada, estriada o perforada-equinulada).

Los géneros Calceolaria y Jovellana de la actual familia Calceolariaceae analizados presentan el mismo tipo polínico, pequeños $(15 \times 11$ $\mu \mathrm{m})$, prolato-esferoidales, 3-colporados, exina semitectada reticulada, en Calceolaria elatior los muros de los retículos son redondeados y en Jovellana violacea, más bien agudos (Figs. 1 A-C, 2 A-D).

Las especies pertenecientes a los géneros Lindernia y Torenia de la familia Linderniaceae tienen granos de polen pequeños en L. dubia (15 x $15 \mu \mathrm{m})$ a medianos en $T$. thouarsii $(28 \times 28 \mu \mathrm{m})$, esferoidales, 3-colpados (T. thouarsii), 3-colporados (L. dubia), exina semitectada reticulada (L. dubia) y en $T$. thouarsii es retículo-rugulada (Figs. 1 D-I, 2 E-F).

El género Mimulus de la familia Phrymaceae, 
Bol. Soc. Argent. Bot. 51 (2) 2016

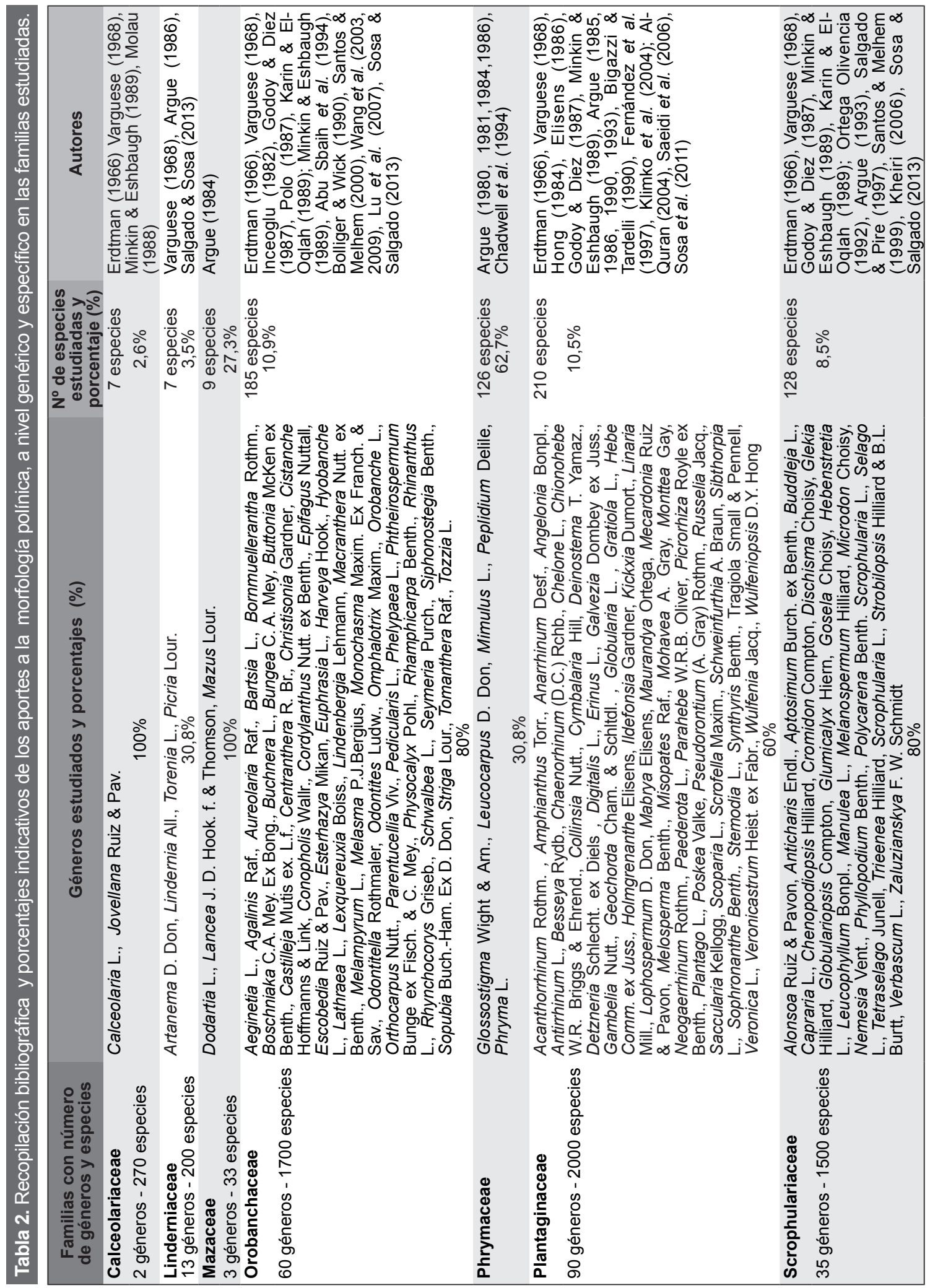




\section{M. Sosa y C. R. Salgado - Palinotaxonomía de Scrophulariaceae sensu lato}

Tabla 3. Lista de caracteres palinológicos

utilizados en el análisis estadístico de las especies de Scrophulariaceae sensu lato.

\begin{tabular}{|c|c|c|}
\hline Variables & Abreviaturas & Codificación \\
\hline Eje polar & $P$ & $\mu \mathrm{m}$ (promedio) \\
\hline Eje ecuatorial & $E$ & $\mu \mathrm{m}$ (promedio) \\
\hline Forma del grano & $\mathrm{P} / \mathrm{E}$ & Valor promedio \\
\hline \multirow{3}{*}{$\begin{array}{l}\text { Estructura } \\
\text { de la exina }\end{array}$} & \multirow{3}{*}{ Est. Ex. } & $0=$ intectado \\
\hline & & $1=$ semitectado \\
\hline & & $2=$ tectado \\
\hline \multirow{15}{*}{$\begin{array}{l}\text { Escultura de } \\
\text { la exina }\end{array}$} & \multirow{15}{*}{ Esc. Ex. } & $0=$ tipo crotonoide \\
\hline & & $1=$ retipilado \\
\hline & & $2=$ reticulado \\
\hline & & $3=$ estriado-perforado \\
\hline & & 4=psilado \\
\hline & & $5=$ foveolado \\
\hline & & $6=$ perforado \\
\hline & & 7=escábrido \\
\hline & & $8=$ rugulado \\
\hline & & $9=$ verrugoso \\
\hline & & $10=$ granulado \\
\hline & & $11=$ estriado \\
\hline & & $12=$ reticulo supratectal \\
\hline & & 13=perforado-escábrido \\
\hline & & $14=$ equinulado \\
\hline \multirow{8}{*}{ Aperturas } & \multirow{8}{*}{ A } & $0=$ inaperturado \\
\hline & & $\begin{array}{l}1=\text { colporado (ora } \\
\text { irregular) }\end{array}$ \\
\hline & & $2=$ colpos \\
\hline & & $3=$ colporados \\
\hline & & 4=diploporados \\
\hline & & $5=e s p i r a p e r t u r a d o s$ \\
\hline & & $6=$ sincolpados \\
\hline & & $7=$ poros \\
\hline $\begin{array}{l}\text { Número de } \\
\text { aperturas }\end{array}$ & NA & Valor numérico \\
\hline
\end{tabular}

presenta granos de polen medianos $(28 \times 28 \mu \mathrm{m})$, esferoidales, espiraperturados (e.g. M. luteus), exina tectada perforada-equinulada (e.g. $M$. glabratus) (Figs. 1 J-L, 3 A-F).
Las especies estudiadas de la familia Orobanchaceae presentan granos medianos (30 x $30 \mu \mathrm{m})$, predominantemente esferoidales, inaperturados (Orobanche lutea), 3-colpados (Seymeria deflexa, Orobanche alba), 4-brevicolpados (Dasistoma macrophyllum), (3) 4-5-colporados (Castilleja lithospermoides). La exina puede ser: semitectada retipilada semejante al patrón-Croton (e.g. Agalinis communis, Melasma melamphyroides), semitectada reticulada heterobrocada (Castilleja lithospermoides) y tectada escábrida-verrugosa (Orobanche minor) (Figs. 4, A-L, 5-6, A-F).

El análisis de las especies en Plantaginaceae muestra granos de polen esferoidales, pequeños (14 x $12 \mu \mathrm{m}$ ) 3-colporados (Mecardonia grandiflora) y pantoporados (7-14 poros en Plantago lanceolata). La exina puede ser: tectada psilada-foveolada (Stemodia lobelioides), perforada (Mecardonia grandiflora) o escábrida (Plantago lanceolata) (Figs. 7 A-F, 8 A-F).

En la familia Scrophulariaceae sensu stricto se describen granos pequeños $(15 \times 15 \mu \mathrm{m})$ a medianos (31 x $31 \mu \mathrm{m})$, esferoidales, 3-colporados (Buddleja grandiflora, Verbascum virgatum), o 3-diploporados en Capraria biflora. La exina puede ser: semitectada reticulada (Verbascum virgatum), tectada psilada (Buddleja), y tectada estriadarugulada (Capraria biflora) (Fig. 7 G-L, 9 A-F).

\section{Análisis estadístico}

El fenograma UPGMA obtenido muestra un coeficiente de correlación cofenética de $\mathrm{r}=0,831$ e indica una buena correlación entre la matriz de los valores cofenéticos y la matriz de distancia taxonómica (Fig. 10). En la Figura 10 se puede observar que la familia Phrymaceae se separa del resto porque es la única que presenta especies con granos de polen espiraperturados, pero también hay 3-4-5-6-7 colpados y 3-colporados; la exina es semitectada reticulada, tectada perforadaequinulada. En otro gran grupo quedan congregadas las restantes familias, las cuales poseen granos de polen colporados. Calceolariaceae se separa de las demás por tener granos de polen pequeños (18 $\mu \mathrm{m}), 3$-colporados y exina semitectada reticulada. La familia Mazaceae posee representantes con granos de polen de tamaño mediano $(25 \mu \mathrm{m})$, todos 3-colporados, poros con márgenes irregulares y exina semitectada reticulada. En el otro grupo 


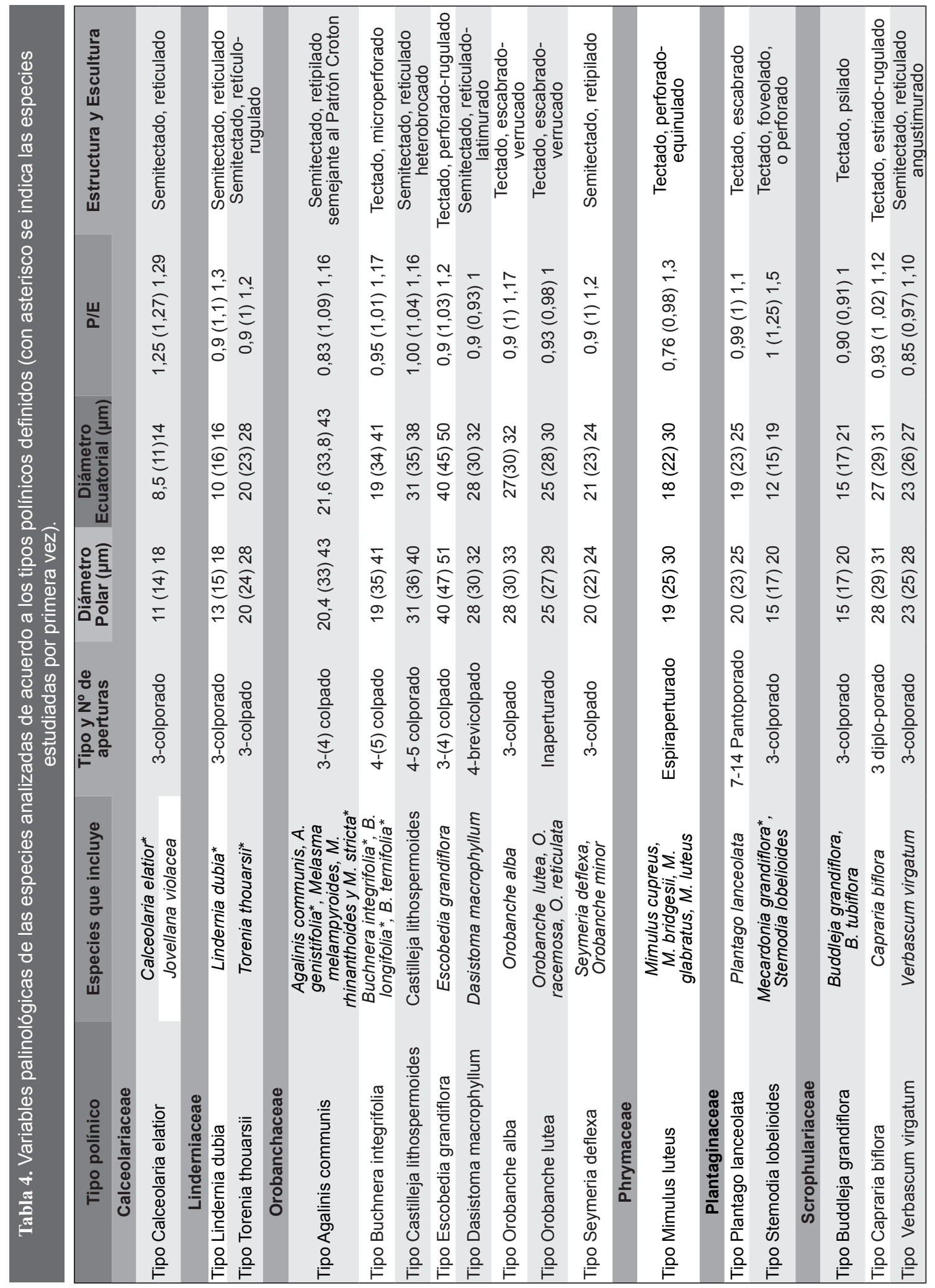


queda Orobanchaceae que se separa de las otras familias por tener, la gran mayoría, granos de polen 3-colpados y exina semitectada retipilada, pero también hay especies con granos inaperturados, sincolpados, 4-colpados y 3-colporados, se destaca el tipo polínico Dasistoma macrophyllum por la forma cuadrangular. La familia Linderniaceae tiene representantes con granos 3-colporados y 3-4 colpados, exina semitectada reticulada, con granos pequeños $(15 \times 15 \mu \mathrm{m})$ a medianos $(30 \times 30 \mu \mathrm{m})$. Finalmente quedan agrupadas Scrophulariaceae y Plantaginaceae, porque ambas tienen representantes con granos de polen 3-colporados y la exina es semitectada reticulada. Scrophulariaceae se diferencia de Plantaginaceae porque la primera presenta granos de polen 3-diploporados y la segunda tiene granos de polen pantoporados, 3-colpados, 3-sincolpados.

\section{Discusıón}

Las especies incluidas actualmente en diferentes familias pero que antes pertenecían a Scrophulariaceae sensu lato, mostraron una gran diversidad polínica en coincidencia con otros autores (Varghese, 1968; Karim \& El-Oqlah, 1989; Inceoglu, 1982; Hong, 1984; Argue, 1980, 1981, 1984, 1985, 1986, 1990, 1993; Bigazzi \& Tardelli, 1990; Correa et al., 1995; Fernández et al., 1997; Santos \& Melhem, 1999, 2000; Wang et al., 2003, 2009; Saeidi-Mehrvarz \& Zarrei, 2006; Kheiri et al., 2006). Las variaciones morfológicas evidenciadas en los 17 tipos polínicos descriptos en el presente estudio; ponen de manifiesto el carácter euripolínico del grupo, destacado por Varghese (1968). Los caracteres polínicos con mayor peso para la clasificación de los distintos grupos taxonómicos fueron: el tipo de apertura, la estructura y el diseño de la escultura de la exina, en coincidencia con Nilsson \& Praglowski (1992).

Scrophulariaceae en su circunscripción tradicional comprendía 306 géneros y cerca de 5850 especies de distribución cosmopolita (Fischer, 2004). Los estudios de filogenia molecular demostraron que la familia es polifilética, lo que llevó a su reestructuración con movimientos de géneros y tribus a otras familias existentes como Plantaginaceae, Orobanchaceae y la elevación de algunas tribus a nivel de familia como Linderniaceae y Calceolariaceae. En ese sentido, Scrophulariaceae sensu stricto se reduce a 35 géneros y 1500 especies, de distribución cosmopolita (Souza \& Lorenzi, 2008). A partir del análisis polínico de 4 especies analizadas en Scrophulariaceae sensu stricto se determinaron 3 tipos polínicos muy diferentes. Estos resultados, sumados a las contribuciones de Erdtman (1966), Varguese (1968), Godoy \& Diez (1987), Minkin \& Eshbaugh (1989), Karim \& ElOqlah (1989), Ortega-Olivencia \& Devesa-Alcaraz (1992), Argue (1993), Salgado \& Pire (1997), Santos \& Melhem (1999), Kheiri et al. (2006), Sosa \& Salgado (2013b), muestran la heterogeneidad polínica de la familia. Si bien la mayoría de las especies se dispersan a través de mónades, en Anticharis linearis (Benth.) Hochst. ex Asch. se registraron tétrades tetraédricas y decusadas, raramente díadas (Varguese, 1968). El tipo polínico predominante en la familia es el 3-colporado, se destaca particularmente el tipo 3-diploporado en Capraria biflora.

Calceolaria y Jovellana son dos géneros que anteriormente se encontraban en la tribu Calceolarieae (Scrophulariaceae); sin embargo los estudios filogenéticos realizados en los últimos tiempos demostraron que la tribu forma un grupo monofilético, razón por la cual proponen elevarla al rango de familia (Andersson, 2006). De esta forma Calceolariaceae está conformada por los dos géneros anteriormente mencionados y aproximadamente 270 especies distribuidas en América, desde México hasta el Sur de Argentina, y algunas especies de Jovellana que crecen en Nueva Zelanda (Molau, 1988; Souza \& Lorenzi, 2008). Las especies analizadas palinológicamente de ambos géneros fueron incluidas en un solo tipo polínico, debido a la uniformidad morfológica que poseen; en coincidencia con lo observado por Erdtman (1966), Varguese (1968), Molau (1988) y Minkin \& Eshbaugh (1989). La homogeneidad palinomorfológica encontrada que ya fue destacada por Molau (1988); podría reforzar la hipótesis del origen monofilético de esta nueva familia.

La familia Phrymaceae originalmente era monogenérica, estaba integrada solamente por Phryma (Cantino, 2004), no obstante a partir de los estudios de filogenia molecular se incorporaron algunos géneros de las tribus Mimuleae (Diplacus, Erythranthe, Mimetanthe, Mimulus), Microcarpeae 
Bol. Soc. Argent. Bot. 51 (2) 2016
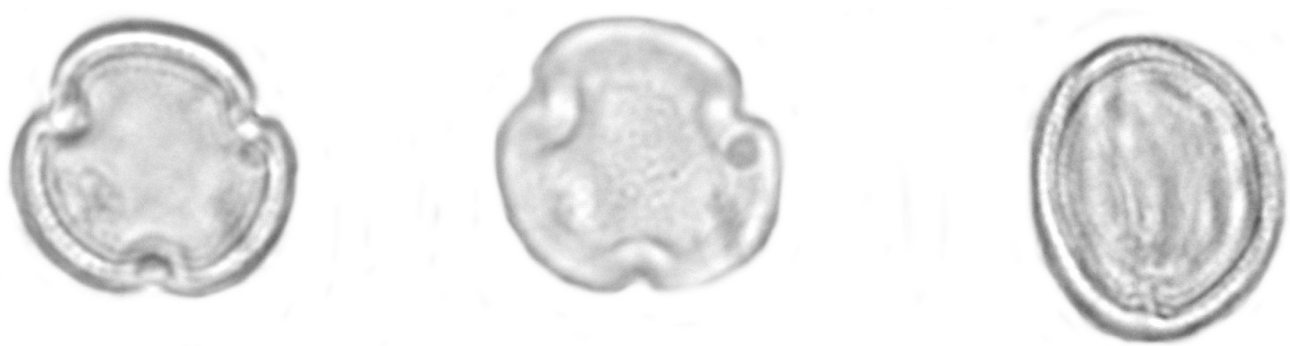

A

B
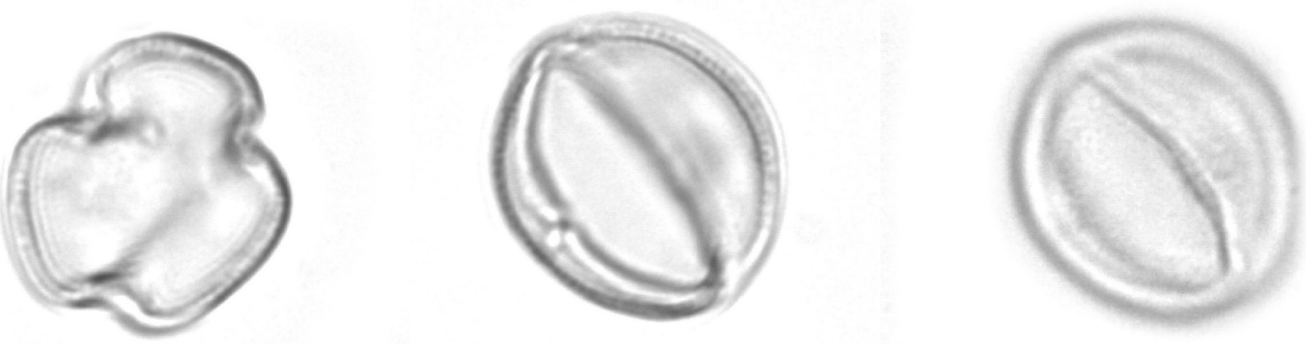

D

E
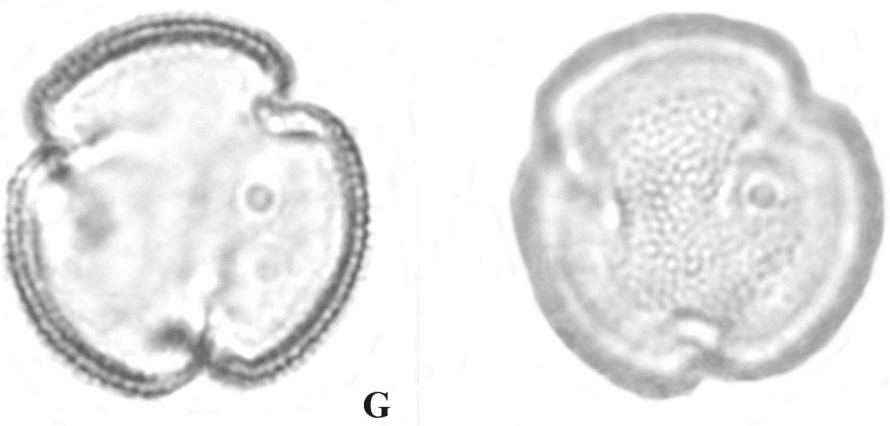

H
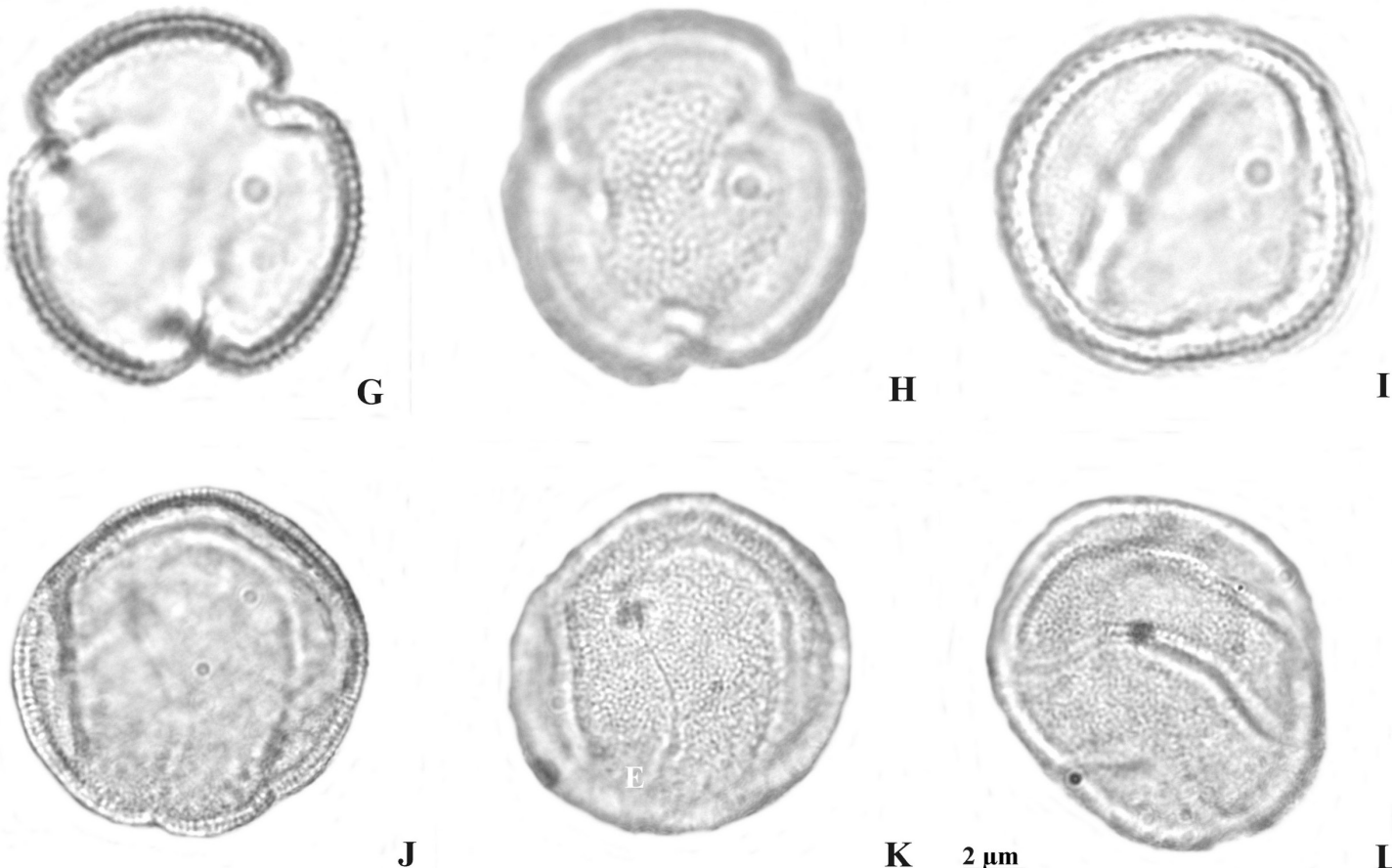

K

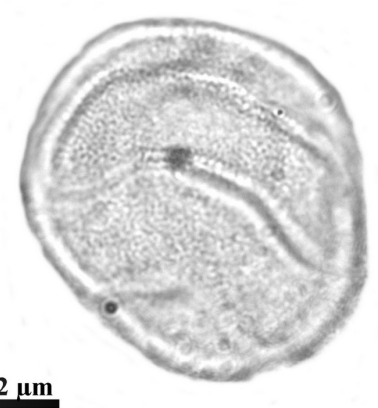

$\mathbf{L}$

Fig. 1. Granos de polen de Calceolariaceae, Linderniaceae y Phrymaceae (MO). A-C: Jovellana violacea. A: vista polar en corte óptico, B: vista polar en foco superior, C: vista ecuatorial en corte óptico. D-F: Lindernia dubia. D: vista polar en corte óptico, E: vista ecuatorial en corte óptico, F: vista ecuatorial en foco superior. G-I: Torenia thouarsii. G: vista polar en corte óptico, H: vista polar en foco superior, I: vista ecuatorial en corte óptico. J-L: Mimulus luteus. J: corte óptico, J-K: foco superior. 

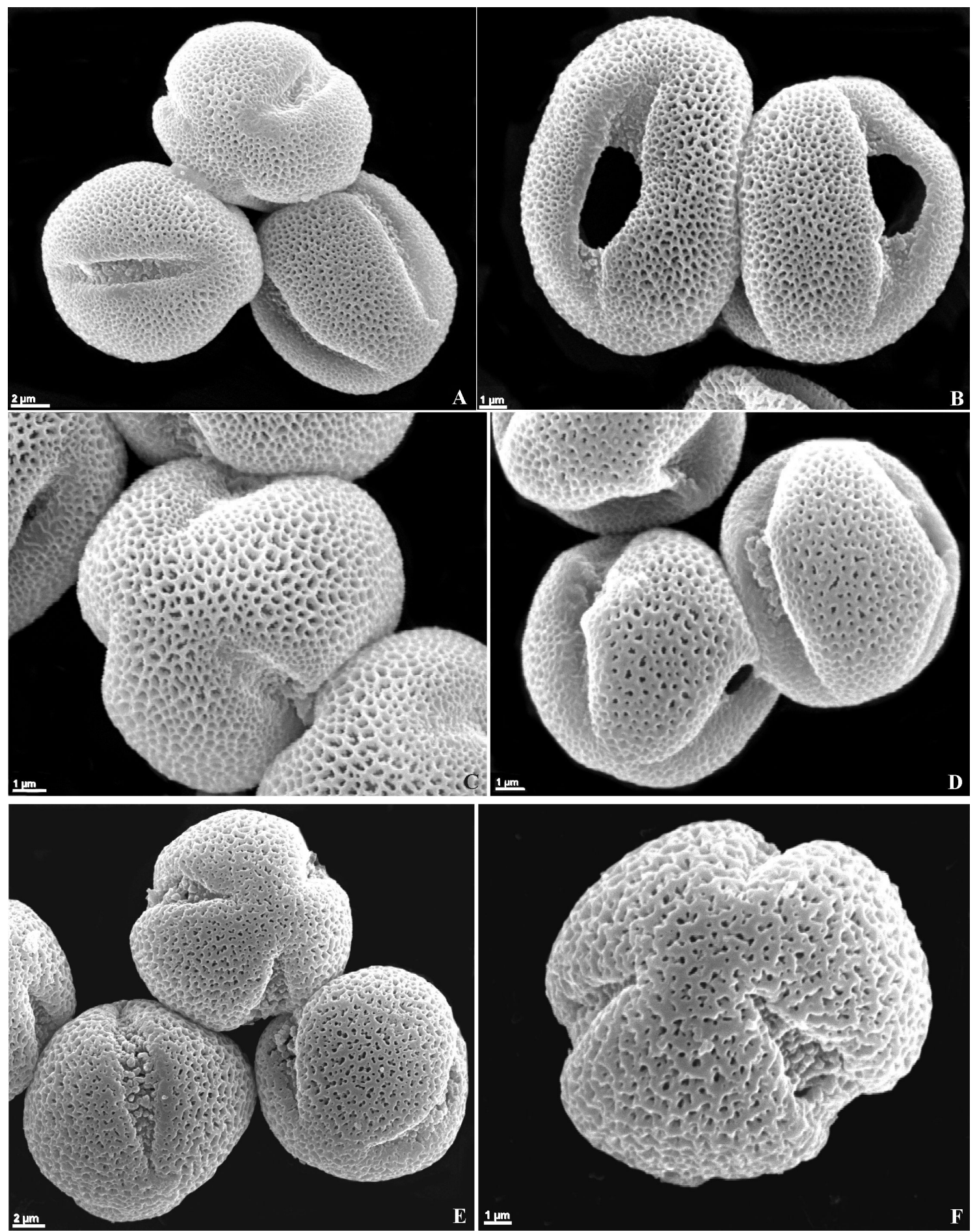

Fig. 2. Granos de polen de Calceolariaceae y Linderniaceae (MEB). A-C: Jovellana violacea. A: vistas polar, subpolar y ecuatorial, B: vistas ecuatoriales, C: vista polar. D: Calceolaria elatior, vistas ecuatoriales. E: Lindernia dubia, vistas ecuatorial y polar. F: Torenia thouarsii, vista polar. 
Bol. Soc. Argent. Bot. 51 (2) 2016
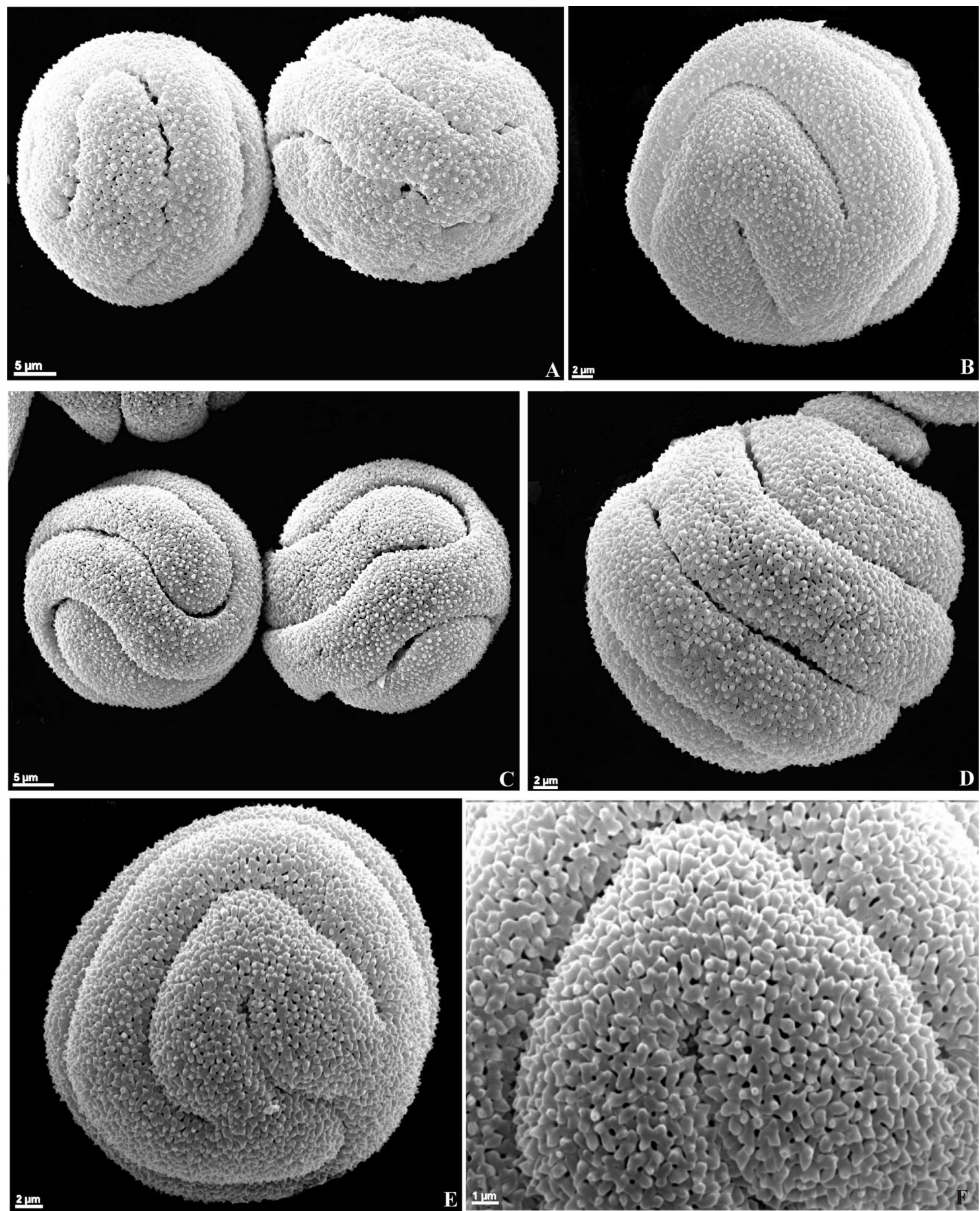

Fig. 3. Granos de polen de Phrymaceae (MEB). A: Mimulus cupreus, vista general. B: M. glabratus, vista polar. C-D: M. luteus. C: vistas generales, D: vista ecuatorial. E-F: M. bridgesii. E: vista polar, F: detalle de la exina. 

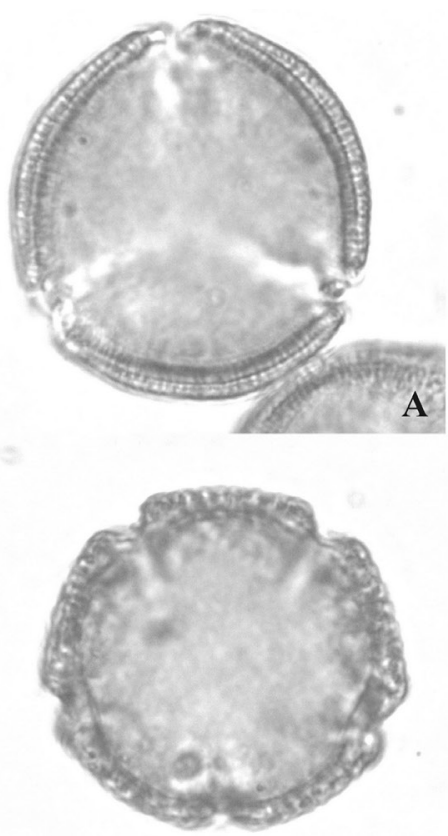

D

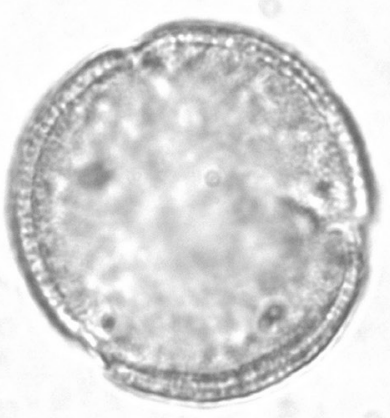

G

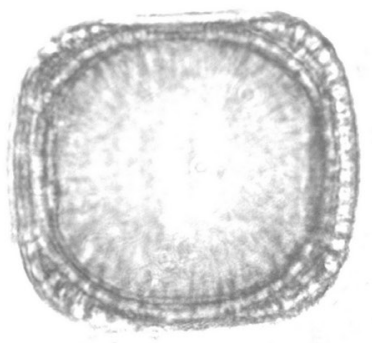

$\mathbf{J}$
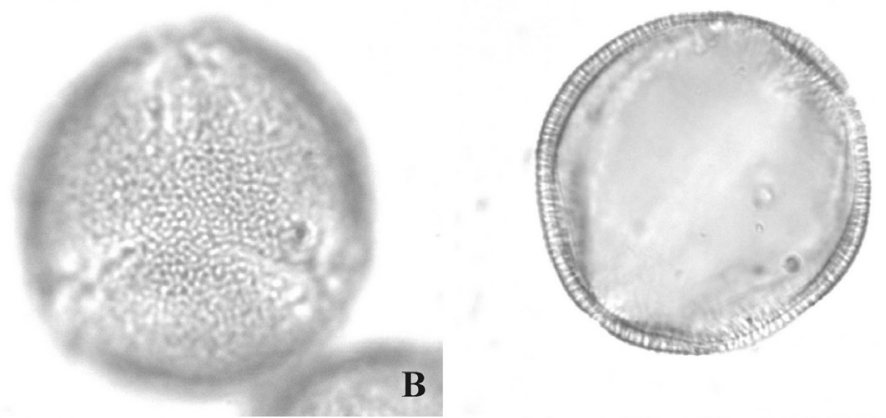

C

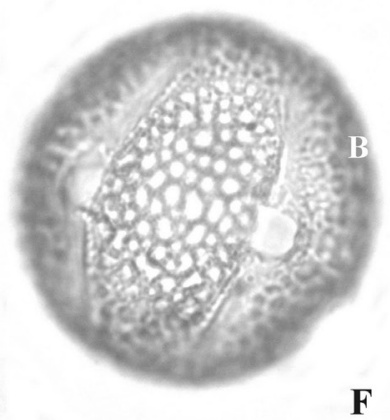

$\mathbf{E}$
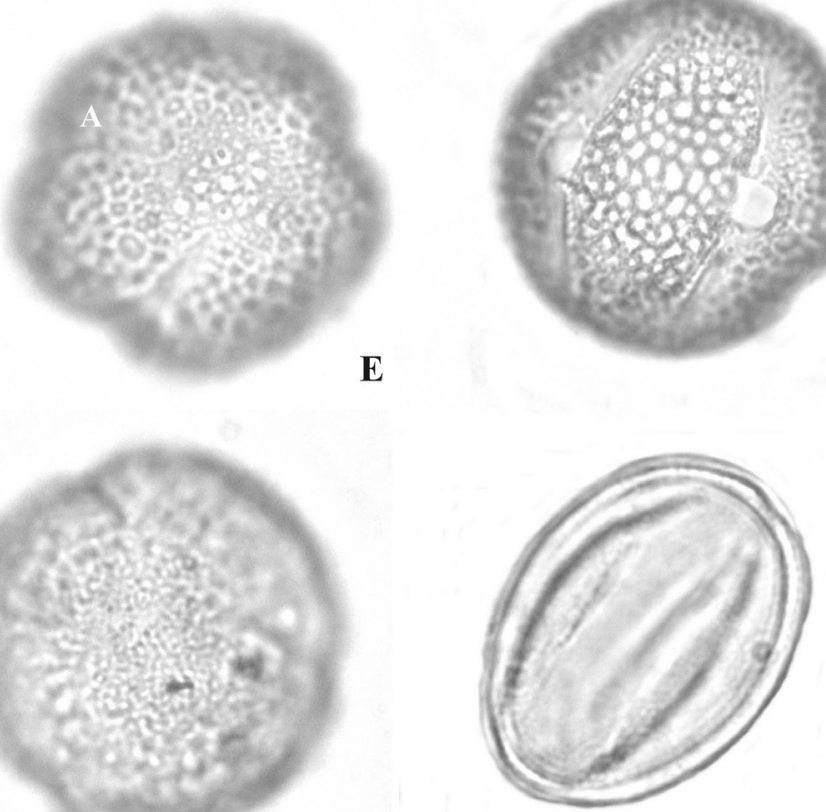

H

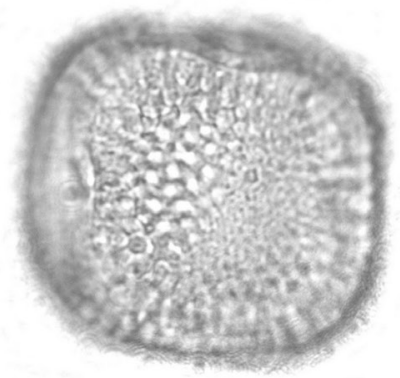

K

K $\quad 2 \mu \mathrm{m}$

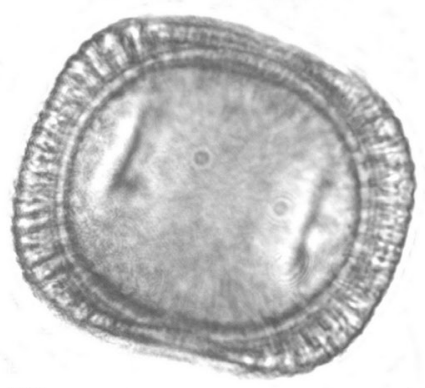

$\mathbf{L}$

Fig. 4. Granos de polen de Orobanchaceae (MO). A-B: Melasma rhinanthoides. A: vista polar en corte óptico, B: vista polar en foco superior. C: Agalinis genistifolia, vista ecuatorial en corte óptico. D-F: Castilleja lithospermoides. D: vista polar en corte óptico, E: vista polar en foco superior, F: vista ecuatorial en foco superior. G-H: Escobedia grandiflora. G: vista polar en corte óptico, H: vista polar en foco superior. I: Orobanche alba, vista polar en corte óptico. J-L: Dasistoma macrophyllum. J: vista polar en corte óptico, K: vista polar en foco superior, L: vista ecuatorial en corte óptico. 
Bol. Soc. Argent. Bot. 51 (2) 2016
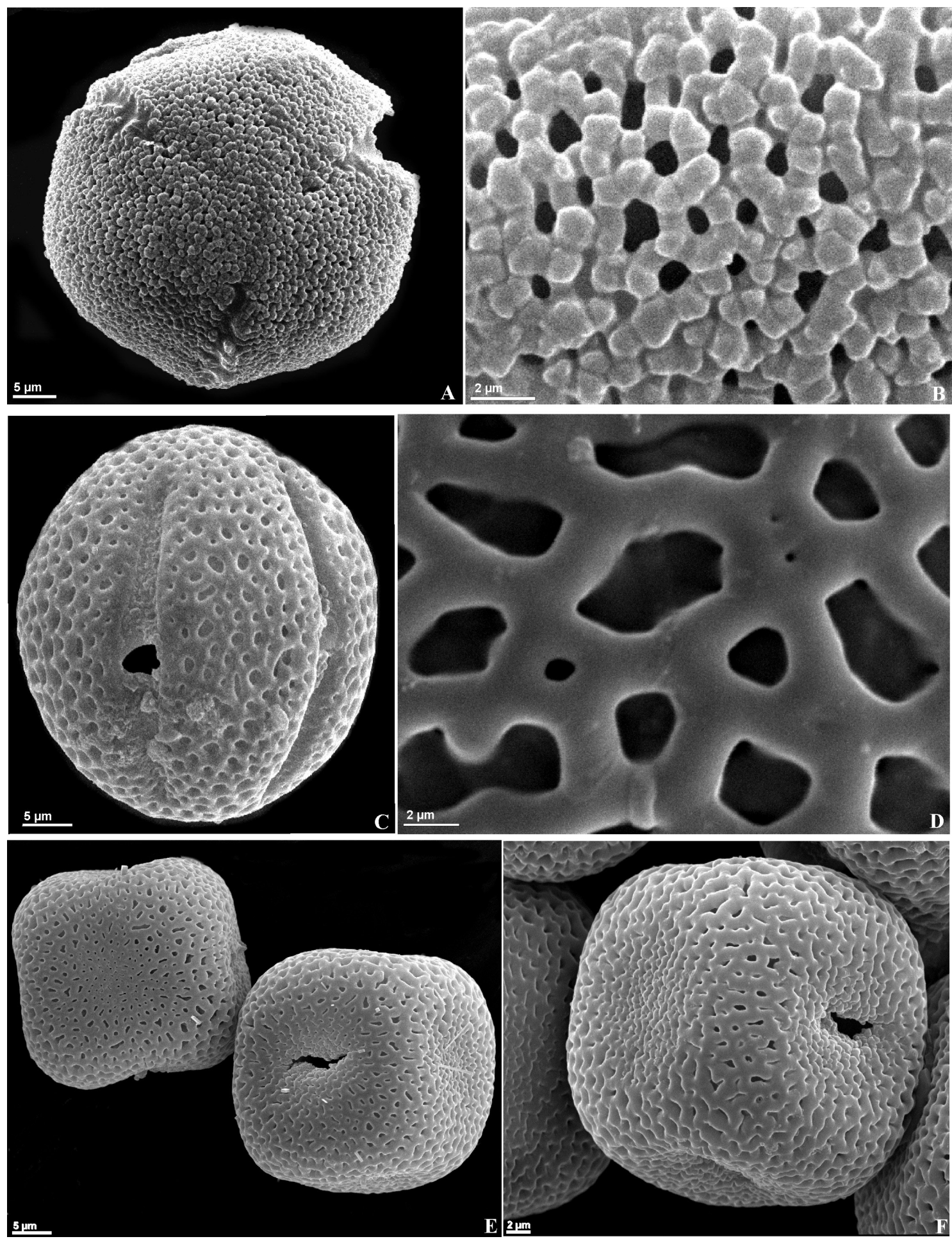

Fig. 5. Granos de polen de Orobanchaceae (MEB). A-B: Melasma rhinanthoides. A, vista polar, B: detalle de la exina. C-D: Castilleja lithospermoides. C: vista ecuatorial, D: detalle de la exina. E-F: Dasistoma macrophyllum. E: vistas polar y subecuatorial y F: vista subpolar. 

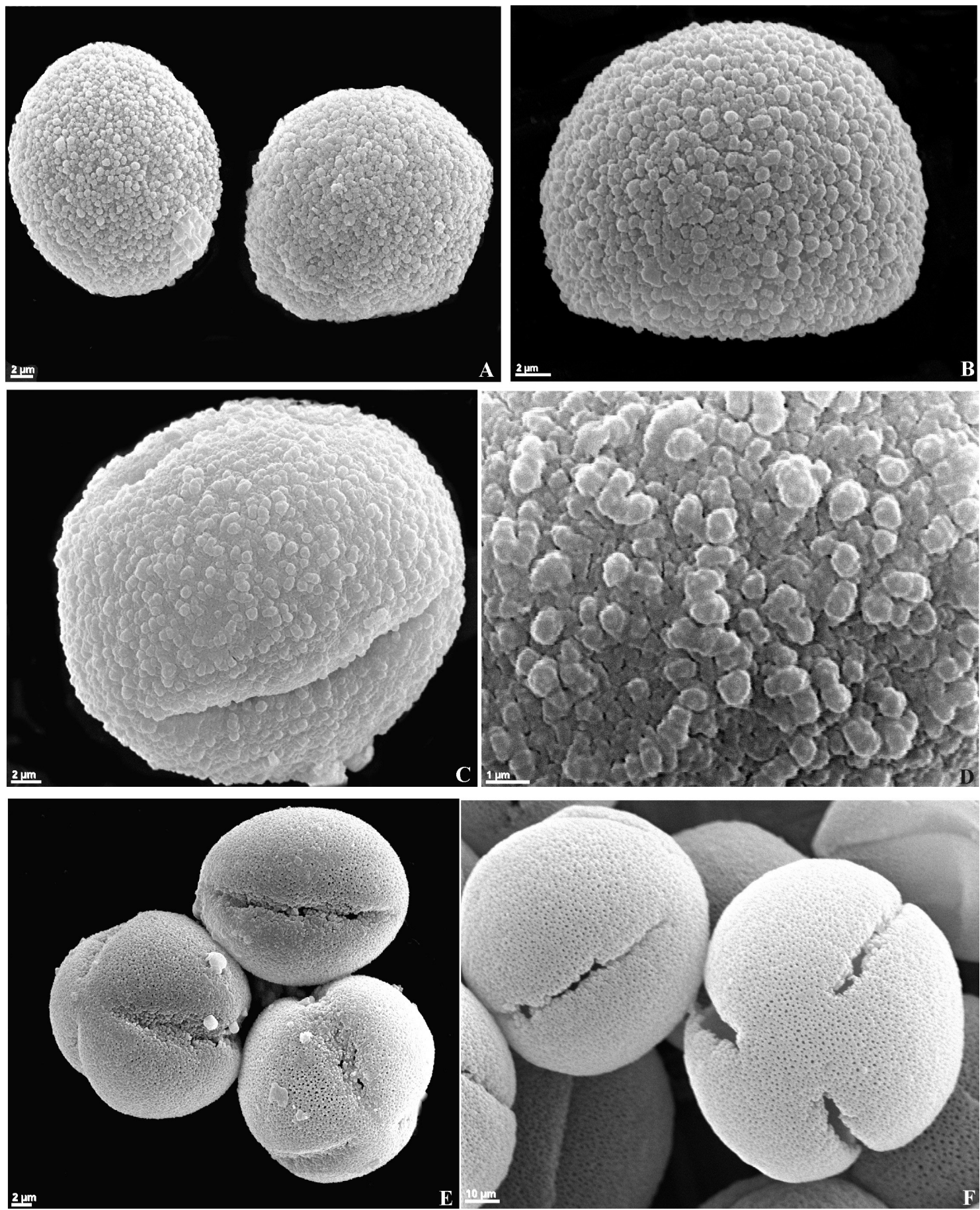

Fig. 6. Granos de polen de Orobanchaceae (MEB). A-B: Orobanche lutea, vistas generales. C-D: O. alba. C: vista ecuatorial, D: detalle de la exina. E: O. minor, vistas polares y ecuatorial. F: Seymeria deflexa, vistas polar y subecuatorial. 

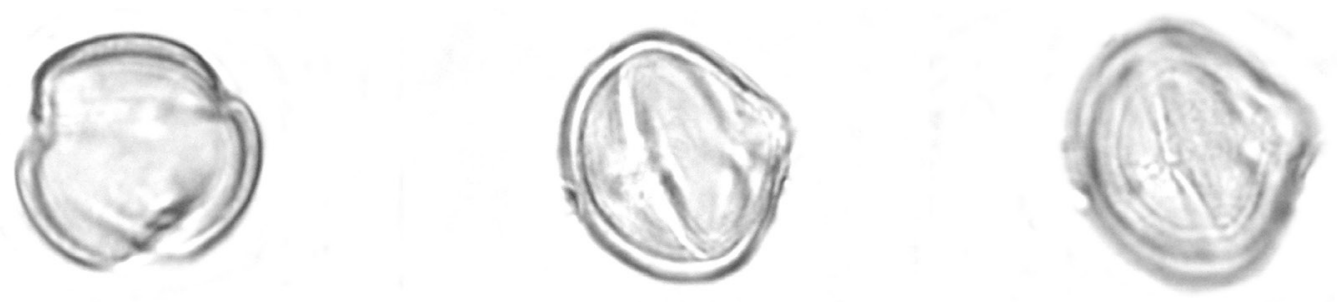

A

B

C
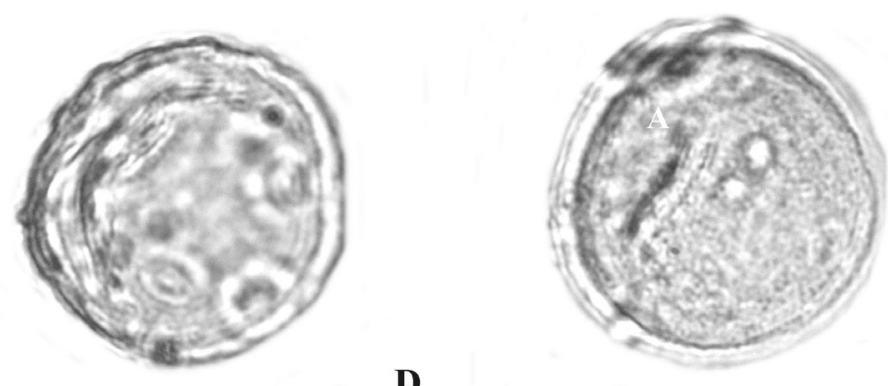

$\mathbf{E}$
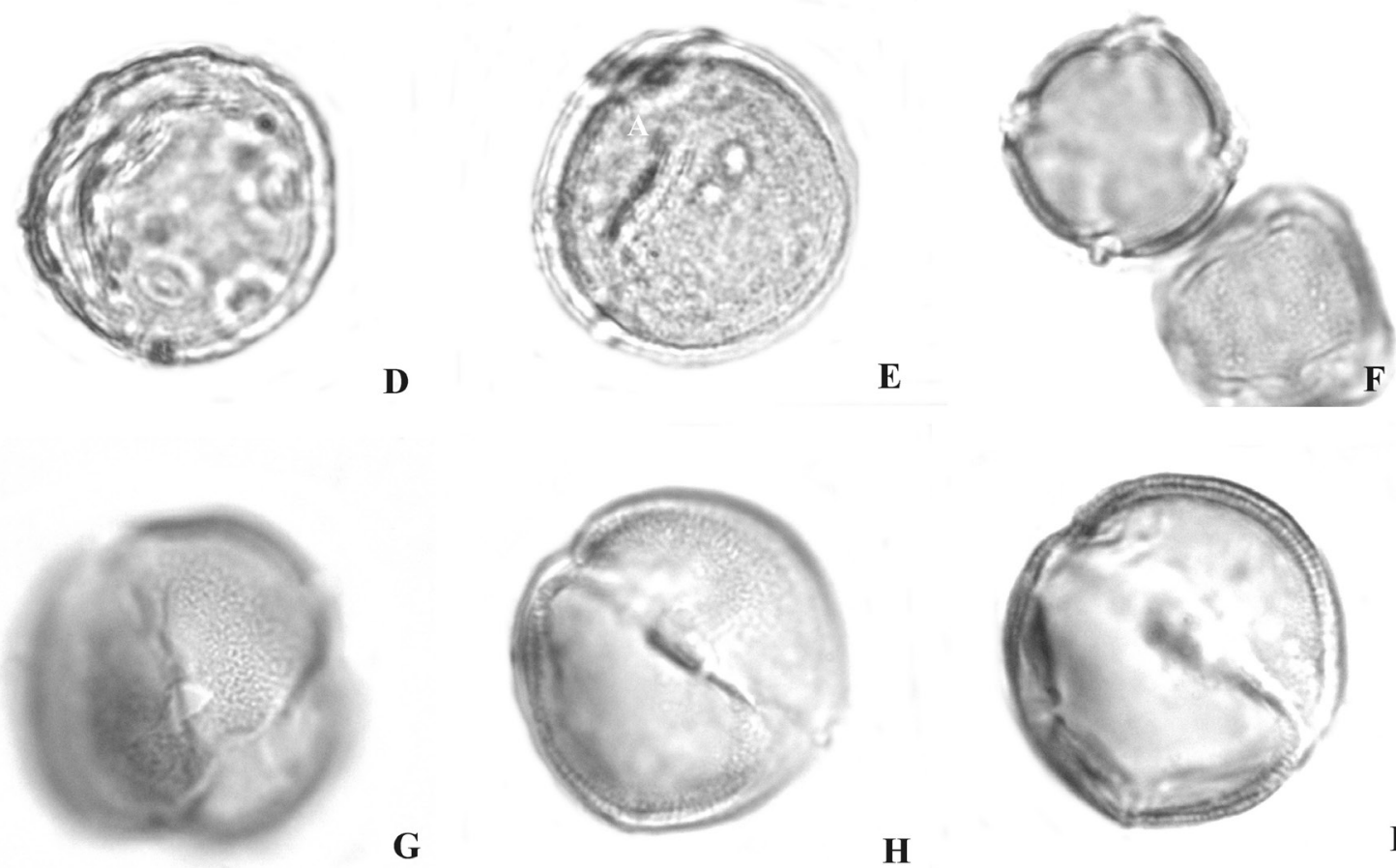

H
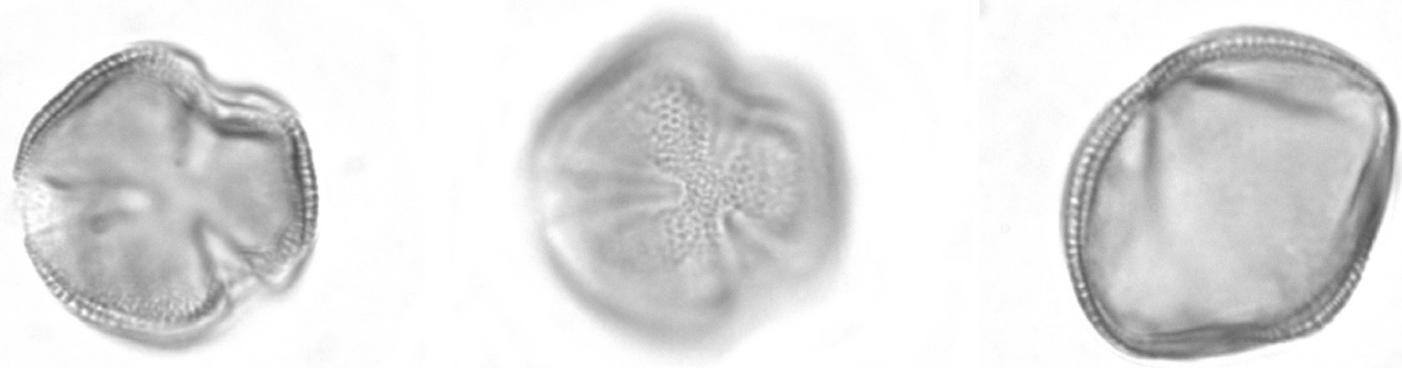

$\mathbf{J}$

K $\quad 2 \mu \mathrm{m}$

$\mathbf{L}$

Fig. 7. Granos de polen de Plantaginaceae y Scrophulariaceae (MO). A-C: Mecardonia grandiflora. A: vista polar en corte óptico, B: vista ecuatorial en corte óptico, C: vista ecuatorial en foco superior. D-E: Plantago australis. D: vista general en corte óptico, E: vista general en foco superior. F: Stemodia lobelioides, vistas polares en corte óptico y foco superior. G-I: Capraria biflora. G: vista polar en corte óptico, H: vista ecuatorial en corte óptico, I: vista ecuatorial en foco superior. J-L: Verbascum virgatum. J: vista polar en corte óptico, $\mathrm{K}$ : vista polar en foco superior, L: vista ecuatorial en corte óptico. 
M. M. Sosa y C. R. Salgado - Palinotaxonomía de Scrophulariaceae sensu lato
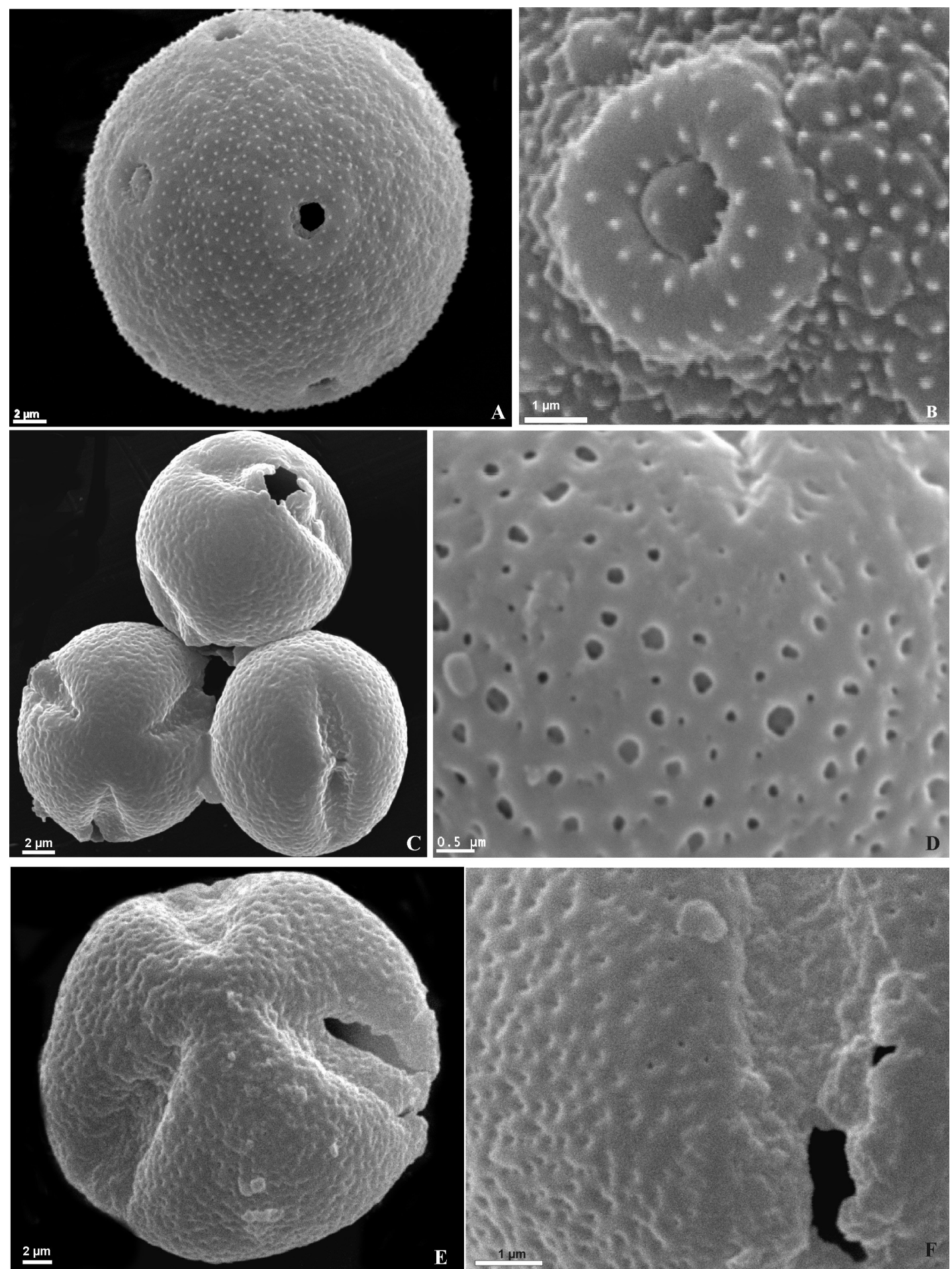

Fig. 8. Granos de polen de Plantaginaceae (MEB). A-B: Plantago lanceolata. A: vista general, B: detalle de la apertura. C-D: Mecardonia grandiflora. C: vistas ecuatoriales y polar, D: detalle de la exina. E-F: Stemodia lobelioides. E: vista polar, F: detalle de la exina y apertura. 
Bol. Soc. Argent. Bot. 51 (2) 2016
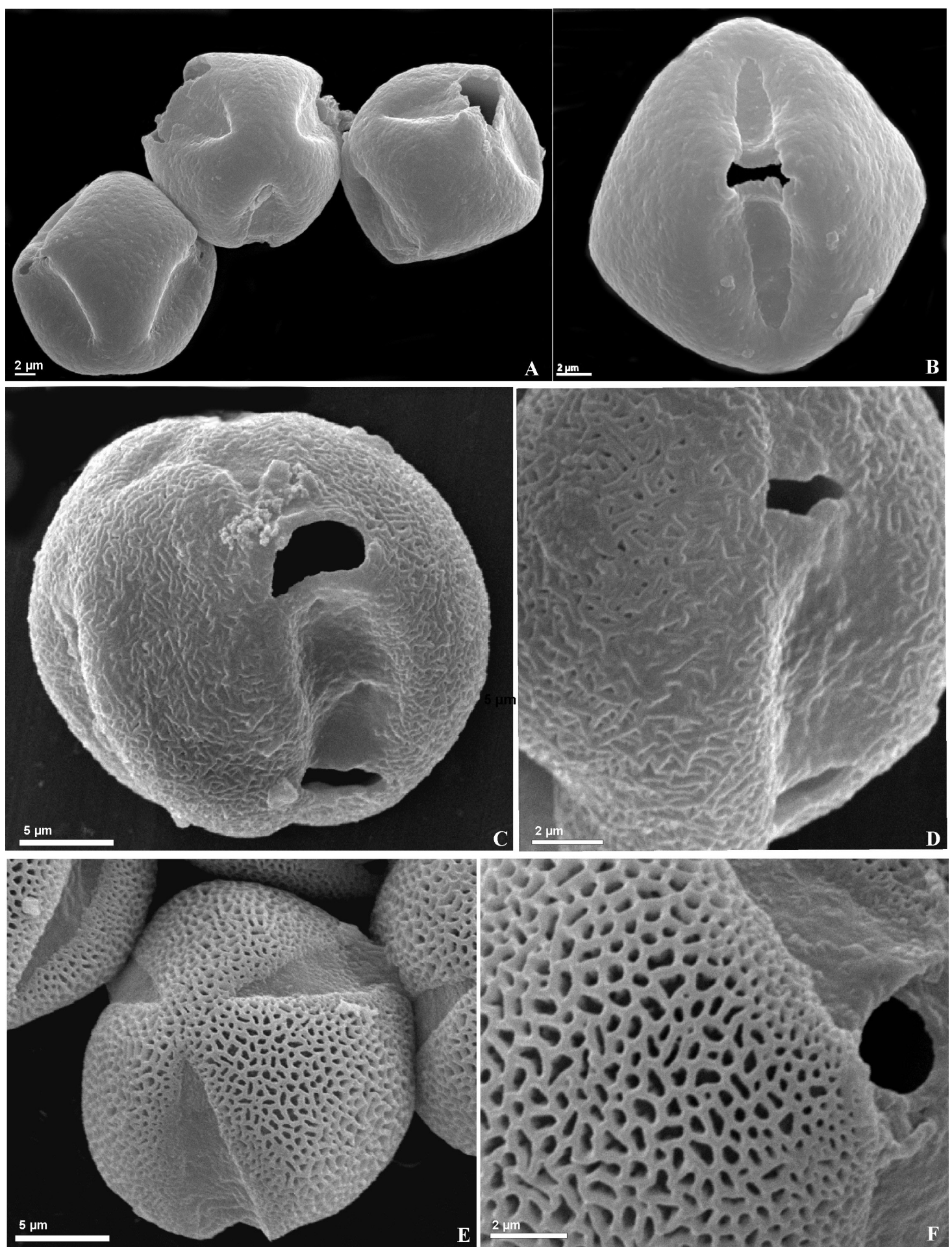

Fig. 9. Granos de polen de Scrophulariaceae (MEB). A: Buddleja tubiflora, vistas polar y ecuatorial. B: B. grandiflora, vista ecuatorial. C-D: Capraria biflora. C: vista ecuatorial, D: detalle de la apertura. E-F: Verbascum virgatum. E: vista polar, F: detalle de la exina. 


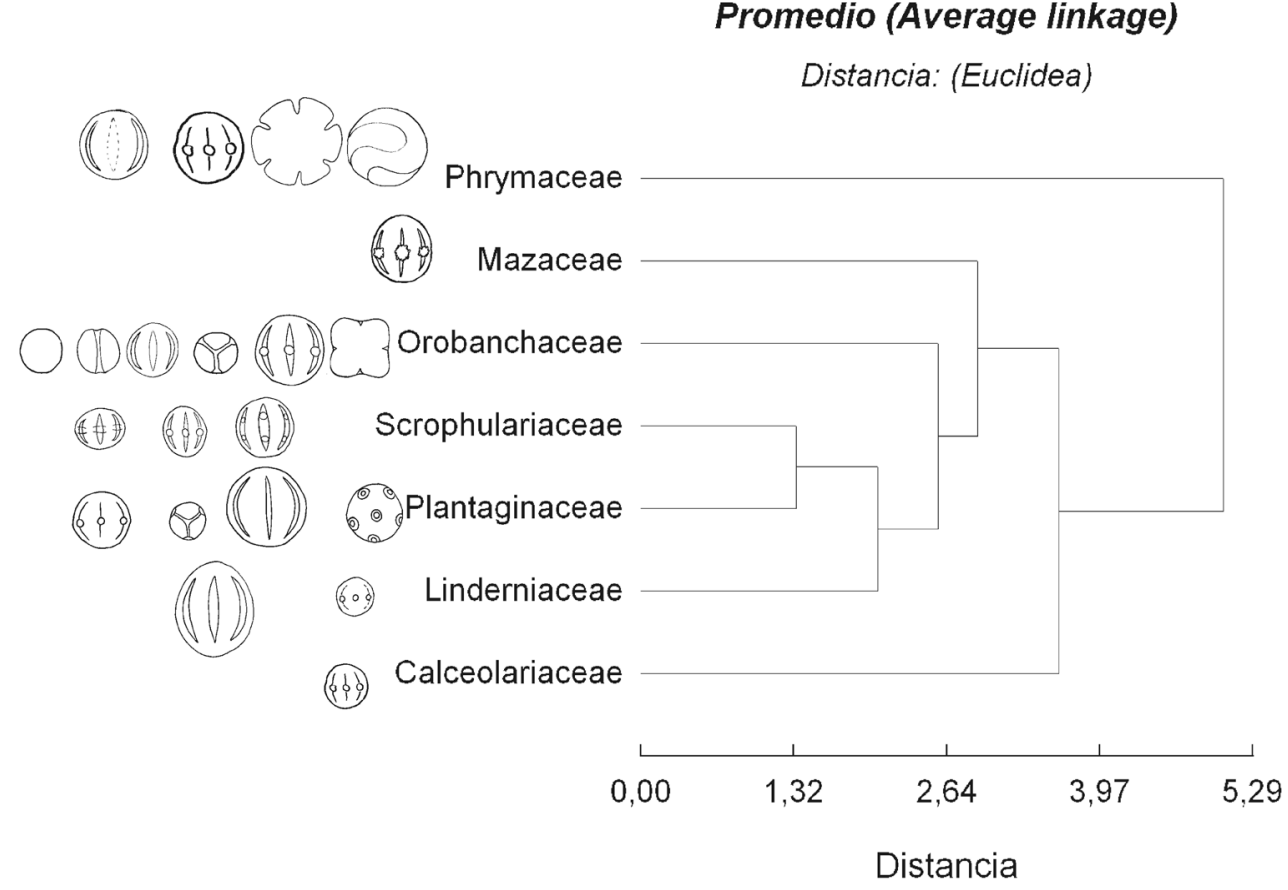

Fig. 10. Fenograma basado en caracteres palinomorfológicos con esquemas de los distintos tipos de granos presentes en cada familia.

(Microcarpaea, Elacholoma, Peplidium, Glossostigma) Leucocarpeae (Hemichaena, Leucocarpus) que pertenecían a Scrophulariaceae sensu lato porque se reunieron en un clado(Beardsley \& Olmstead, 2002). De esta manera, la familia Phrymaceae queda representada con 13 géneros y aproximadamente 188 especies de distribución amplia a nivel mundial, especialmente en los trópicos húmedos y templados en Norteamérica, Australia y algunas especies de Asia (Stevens, 2001, onwards). Los resultados de nuestros análisis realizados en especies de Mimulus definen un solo tipo polínico: espiraperturado. Sin embargo, en este género, Argue (1980, 1981, 1984 y 1986) determinó varios tipos polínicos en base a las aperturas: 3-colpado, 3-colporado, estéfanocolpado y espiraperturado, con varios subtipos de acuerdo a la escultura y tamaño. Esta variabilidad polínica observada en el género podría relacionarse con la hipótesis de su origen no monofilético (Beardsley \& Olmstead, 2002). El tipo polínico 3-colpado también se presenta en Glossostigma, Phryma y
Peplidium y 3-colporado en Leucocarpus (Argue, 1984, 1986, Chadwell et al., 1992); razón por la cual se consideraría filogenéticamente a estos géneros como derivados de Mimulus (Beardsley \& Barker, 2005).

Los géneros Dodartia, Lancea y Mazus estaban incluidos en la tribu Mimuleae-Scrophulariaceae (Fischer, 2004). Actualmente estos géneros son tratados como integrantes de la familia Mazaceae de acuerdo a los análisis de filogenia (Beardsley \& Olmstead, 2002); de esa manera, estaría conformada por 33 especies, las cuales la gran mayoría pertenecen al género Mazus. Se conoce la morfología polínica de los tres géneros, quienes presentan uniformidad en los caracteres polínicos; de acuerdo a Argue (1984). Esta familia está filogenéticamente emparentada con Phrymaceae (Beardsley \& Olmstead, 2002), ya que géneros de ambas familias estaban dentro de la misma tribu (Mimuleae-Scrophulariaceae). En ese aspecto las dos familias comparten características polínicas, ya que tienen granos de polen 3-colporados, la 
diferencia radica en que los géneros de Mazaceae estudiados poseen endoapertura con márgenes irregulares.

Los géneros de la tribu Lindernieae (Scrophulariaceae), como Lindernia y Torenia están estrechamente relacionadas con los representantes de la tribu Gratioleae (Scrophulariaceae), porque comparten algunas características morfológicas, de esta manera Wettstein (1897) los había incluido dentro de Gratioleae. Los estudios filogenéticos realizados demostraron que ambas tribus Gratioleae y Lindernieae forman dos linajes independientes, razón por la cual proponen elevar al rango de familia a esta última (Rahmanzadeh et al., 2005). De acuerdo a ello, la familia Linderniaceae incluye actualmente 13 géneros y cerca de 200 especies, distribuidas en regiones tropicales y templadas de África, SE de Asia, América del Norte y América del Sur (Souza \& Lorenzi, 2008). Las descripciones de los granos de polen de dos especies de Lindernia y Torenia analizados permitieron definir dos tipos polínicos; estos resultados coinciden con los aportes realizados por Varguese (1968), Argue (1986), Roubik \& Moreno (1991), Lewis (2000) y Salgado \& Sosa (2013). Desde el punto de vista palinológico las especies que actualmente se encuentran dentro de la tribu Gratioleae (Plantaginaceae), como Mecardonia y Stemodia, comparten las características polínicas con el género Lindernia, ya que son pequeñas y 3-colporadas; sin embargo Torenia es 3-colpada como Veronica (Plantaginaceae).

La familia Plantaginaceae originalmente incluía solamente al género Plantago (Schwarzbach, 2004). En las dos últimas décadas, los estudios de filogenia han reestructurado la familia, incorporando géneros de varias tribus de Scrophulariaceae sensu lato como Angelonieae, Antirrhineae, Callitricheae, Cheloneae, Digitalideae, Gratiolae, Globularieae, Russelieae, Sibthorpieae y Veroniceae (Albach et al., 2005). Actualmente, esta familia de distribución cosmopolita, cuenta con aproximadamente 90 géneros y más de 2000 especies (Stevens, 2001, onwards). Debido a que esta familia es considerada altamente hetereogénea, porque incluye a gran cantidad de géneros que habitan diferentes ambientes, su circunscripción morfológica es muy somera (Albach et al., 2005). Los resultados palinológicos obtenidos a partir de las especies de Plantago, Mecardonia y Stemodia (Plantaginaceae) estudiadas permitieron definir dos tipos polínicos, demostrando la heterogeneidad en su morfología en coincidencia con Erdtman (1966), Varguese (1968) Hong (1984), Elisens (1986), Godoy \& Díez (1987), Minkin \& Eshbaugh (1989), Argue (1985, 1986, 1990, 1993), Candau (1987), Bigazzi \& Tardelli (1990), Fernández et al. (1997), Al-Quran (2004), Klimko et al. (2004), Saeidi-Mehrvarz \& Zarrei (2006) y Sosa et al. (2012). Las especies del género Plantago, presentan granos de polen pantoporados; que se pueden diferenciar respecto del tamaño y número de poros, presencia o ausencia de anillos y opérculos (Al-Qurán, 2004; Klimko et al., 2004). La gran mayoría de las especies analizadas en el resto de la familia poseen granos 3-colporados (Stemodia, Gratiola, Linaria, Mecardonia), y algunas 3-colpadas (Chelone, Veronica). En la tribu Veroniceae se describen granos de polen 3-colpados y 3-colporados, como la escultura es variable permitió determinar varios tipos polínicos, carácter que contribuyó a separar en secciones o grupos a la tribu y al género Veronica (Hong, 1984; Fernández et al., 1997; Saeidi-Mehrvarz \& Zarrei, 2006).

La familia Orobanchaceae fue tradicionalmente circunscripta a géneros holoparásitos como Orobanche, Epifagus y Conopholis (Fischer, 2004). Estudios filogenéticos realizados por Olmstead et al. (2001) definieron un clado monofilético que incluye especies hemiparásitas (tribu Rhinantheae -Scrophulariaceae) y especies de Orobanchaceae. Estos resultados permitieron incorporar las especies hemiparásitas y holoparásitas dentro de la familia Orobanchaceae (Bennett \& Mathews, 2006). De esa manera la actual familia queda constituida por 60 géneros y más de 1700 especies, de distribución cosmopolita (Souza \& Lorenzi, 2008). En las diecisiete especies analizadas desde el punto de vista polínico, pertenecientes a ocho géneros de la familia, se diferenciaron 8 morfotipos. Esta variabilidad palinológica coincide con lo descripto por Varghese (1968); Inceoglu (1982); Godoy \& Díez (1987); Polo (1987); Karim \& El-Oqlah (1989); Minkin \& Eshbaugh (1989); Bolliger \& Wick (1990); Abu Sbaih et al. (1994); Santos \& Melhem (2000), Wang et al. (2003, 2009); Lu et al. (2007), Sosa \& Salgado (2013a) y Zare et al. (2014). La alta heterogeneidad palinológica del grupo estaría avalando la hipótesis del carácter no monofilético del grupo (Lu et al., 2007). El tipo polínico más frecuente en la familia es el 3-colpado semitectado retipilado, carácter 


\section{M. Sosa y C. R. Salgado - Palinotaxonomía de Scrophulariaceae sensu lato}

palinomorfológico destacado anteriormente por Bennett \& Mathews (2006) como particular en las especies hemiparásitas y holoparásitas.

\section{CONCLUSIONES}

Los resultados indican que Calceolariaceae, Linderniaceae y Mazaceae pueden ser consideradas estenopolínicas, a pesar de que solamente se conoce la morfología polínica de alrededor del 3\% de las especies que integran las dos primeras familias y un $27 \%$ de las especies que integran la tercera familia. En las familias Orobanchaceae, Plantaginaceae y Scrophulariaceae se observó mayor variabilidad polínica por ello se las define como euripolínicas, considerando que se conoce alrededor del $10 \%$ de la morfología del polen. En Phrymaceae se conoce el $62 \%$ de la morfología polínica y también se pudo observar una gran variabilidad lo que determinó su carácter euripolínico.

La heterogeneidad de la morfología polínica (tipo, número de aperturas y diseño de la exina) asociada a otros caracteres morfológicos podrían confirmar la hipótesis del carácter monofilético o polifilético de las diferentes familias analizadas. La variedad de tipos de aperturas, registradas en la totalidad del grupo, podría ser utilizada para establecer posibles líneas evolutivas y confirmar relaciones de parentesco entre las familias estudiadas.

El análisis estadístico realizado en Scrophulariaceae sensu lato, permitió concluir que el polen es un valioso carácter para apoyar la clasificación sistemática actual, sobre todo a nivel de familia y género $(64,4 \%$ de los géneros fueron estudiados). Sin embargo, resulta necesario continuar realizando estudios en el resto de las especies para poder efectuar mayores inferencias a nivel específico, ya que solamente un $11 \%$ de las especies fueron estudiadas desde el punto de vista palinológico.

\section{Agradecimientos}

Agradecemos a Betiana Angulo por su asesoramiento en los análisis estadísticos y a Mirtha Liliana Gómez por pasar a tinta los esquemas. A Cristina Tellería y Sara Tressens por la lectura crítica del manuscrito. Al CONICET y a la SGCyT de la UNNE (PI P002-11) por el apoyo económico para el desarrollo de este trabajo.

\section{Bibliografía}

ABU-SBAIH, H., D. M. KEITH-LUCAS \& S. L.JURY. 1994. Pollen morphology of the genus Orobanche L. (Orobanchaceae). Bot. J. Linn. Soc. 116: 305-313.

ALBACH, D. C., H. M. MEUDT \& B. OXELMAN. 2005. Piecing together the "new" Plantaginaceae. Amer. J. Bot. 92: 297-315.

AL-QURAN, S. A. 2004. Pollen morphology of Plantaginaceae in Jordan. Pakistan J. Biol. Agric. Sci. 7 (9): 1594-1602.

ANDERSSON, S. 2006. On the Phylogeny of the genus Calceolaria (Calceolariaceae) as inferred from ITS and plastid matK sequences. Taxon 55: 125-137.

APG III. 2009. An update of the Angiosperm Phylogeny Group classification for the orders and families of flowering plants: APG III. Bot. J. Linn. Soc. 161: 105-121.

ARGUE, C. L. 1980. Pollen morphology in the genus Mimulus (Scrophulariaceae) and its taxonomic significance. Amer. J. Bot. 67: 68-87.

ARGUE, C. L. 1981. The taxonomic implications of pollen morphology in some South American species of Mimulus (Scrophulariaceae). Amer. J. Bot. 68: 200-205.

ARGUE, C. L. 1984. Pollen morphology in Dodartia, Lancea, Leucocarpus, and Mazus and an analysis of pollen morphotypes in the Mimuleae (Scrophulariaceae). Canad. J. Bot. 61: 53-62.

ARGUE, C. L. 1985. Pollen morphology in the genera Monttea and Melosperma (Scrophulariaceae). Amer. J. Bot. 72: 1248-1255.

ARGUE, C. L. 1986. Pollen morphology of Amphianthus, Artanema, Curanga, Glossostigma, and Peplidium (Scrophulariaceae-Gratioleae). Amer. J. Bot. 73 (11): 1570-1576.

ARGUE, C. L. 1990. Pollen morphology of Deinostema, Geochorda, Gratiola, Ildefonsia, Sophronanthe, and Tragiola (Scrophulariaceae, Gratioleae, Gratiolinae). Canad. J. Bot. 68: 1651-1660.

ARGUE, C. L. 1993. Pollen morphology in the Selagineae, Manuleae (Scrophulariaceae) and selected Globulariaceae and its taxonomic significance. Amer. J. Bot. 80 (6): 723-733.

BEARDSLEY P. M. \& R. G. OLMSTEAD. 2002. Redefining Phrymaceae: the placement of Mimulus, tribe Mimulae, and Phryma. Amer. J. Bot. 89: $1093-$ 1102.

BEARDSLEY, P. M. \& W. R. BARKER. 2005. Patterns of evolution in Australian Mimulus and related genera 
(Phrymaceae $\sim$ Scrophulariaceae): a molecular phylogeny using chloroplast and nuclear sequence data. Austral. Syst. Bot 18: 61-53.

BENNETT, J. R. \& S. MATHEWS. 2006. Phylogeny of the parasitic plant family Orobanchaceae inferred from phytochrome A. Amer. J. Bot. 93 (7): 10391051.

BENTHAM, G. 1846. Scrophulariaceae in De Candolle, Prodromus 10: 186-586.

BENTHAM, G. 1876. In Benthan G. \& J. D. Hooker. Scrophularineae in Genera Plantarum 2: 913-980.

BIGAZZI, M. \& M. TARDELLI. 1990. Pollen morphology and ultra structure of the Old World Antirrhineae (Scrophulariaceae). Grana 29: 257274.

BOLLIGER, M. \& L. WICK. 1990. The pollen morphology of Odontites (Scrophulariaceae) and its taxonomic significance. Pl. Syst. Evol. 173: 159-178.

CANDAU, P. 1987. Plantaginaceae. En Valdés, B.; Díez, M.J. \& I. Fernández. Atlas polínico de Andalucía Occidental. Instituto de Desarrollo Regional Na43, Universidad de Sevilla. EXCMA. Diputación de Cádiz. 293-294.

CANTINO, P. D. 2004. Phrymaceae. In "The families and genera of vascular plants". Ed. K. Kubitzki pp. 323-326- Springer-Berlin.

CHADWELL, T. B., S. J. WAGSTAFF \& P. D. CANTINO. 1992. Pollen morphology of Phryma and some putatives relatives. Syst. Bot. 17: 210-219.

CORREA, A. M. S., M. S. F. SILVESTRE-CAPELETO \& T. MELHEM. 1995. Flora polínica da Reserva do Parque Estadual das Fontes do Ipiranga (São Pablo), Brasil. Familia: 148 - Scrophulariaceae. Hoehnea 22: $109-114$.

CRONQUIST, A. 1981. An integrated system of classification of flowering plants. Columbia University Press: New York.

DI RIENZO, J. A., F. CASANOVES, M. G. BALZARINI, L. GONZALEZ, M. TABLADA \& C. W. ROBLEDO. InfoStat versión 2013. Grupo InfoStat. FCA. Universidad Nacional de Córdoba, Argentina.

ELISENS, W. J. 1986. Pollen morphology and systematic relationships among new world species in tribe Antirrhineae (Scrophulariaceae). Amer. J. Bot. 73: 1298-1311.

ERDTMAN, G. 1960. The acetolysis method. Svensk Botanisk Tidskrskrift 54: 561-564.

ERDTMAN, G. 1966. Pollen morphology and plant taxonomy Angiosperms. Hafner Publ. Co., New York.

FERNÁNDEZ, I., R. JUAN \& J. PASTOR. 1997. Morfología polínica de Veronica L. (Scrophulariaceae) en el Sur oeste de España. Acta Bot. Malac. 22: 65 -72.

FISCHER, E. 2004. Scrophulariaceae. In "The families and genera of vascular plants". Ed. K. Kubitzki pp. 333-432- Springer-Berlin.

GODOY, M. C. \& M. J. DIEZ. 1987. Scrophulariaceae. En Valdéz, B.; Díez, M.J. y I, Fernández. Atlas polínico de Andalucía Occidental. Instituto de Desarrollo Regional Nª3, Universidad de Sevilla. EXCMA. Diputación de Cádiz. 297-307.

HONG, D. 1984. Taxonomy and evolution of the Veroniceae (Scrophulariaceae) with special reference to palynology. Opera Bot. 75: 1-60.

INCEOGLU, O. 1982. Pollen grain in some Turkish Rhinantheae (Scrophulariaceae). Grana 21: 83-96.

KARIM, F. M. \& A. A. EL-OQLAH 1989. Palynological studies in the family Scrophulariaceae from Jordan and Iraq. Pollen et Spores 31: 203-214.

KHEIRI, S., M. KHAYAMI, S. K.OSALOO \& A. MAHMOODZADEH. 2006. Pollen morphology of some species of Verbascum (Scrophulariaceae) in Urmia. Pakistan J. Biol. Agric. Sci. 9: 434-436.

KLIMKO, M., K. IDZIKOWSKA, M. TRUCHAN \& A. KTREFT. 2004. Pollen morphology of Plantago species native to Poland and their taxonomic implications. Acta Soc. Bot. Poloniae 73: 315-325.

LEWIS, D. Q. 2000. A Revision of the New World Species of Lindernia (Scrophulariaceae). Castanea 65: 93-122.

LU, L., H. WANG, S. BLACKMORE, D. Z. LI \& L. N. DONG. 2007. Pollen morphology of the tribe Rhinantheae (Orobanchaceae). Pl. Syst. Evol. 268: 177-198.

MINKIN, J. P. \& W. H. ESHBAUGH. 1989. Pollen morphology of the Orobanchaceae and rhinanthoid Scrophulariaceae. Grana 28: 1-18.

MOLAU, U. 1988. Scrophulariaceae-Part I. Calceolarieae. Flora Neotropica. Monograph 47: 325 pp.

NILSSON, S. \& L. PRAGLOWSKI. 1992.Erdtman 's Handbook of Palynology. Segunda edición. 580pp. Munksgaard. ISBN 87-16-10846-9.

OLMSTEAD, R. G. \& P. A. REEVES. 1995. Evidence for polyphyly of the Scrophulariaceae based on chloroplast $r b c l$ and $n d h F$ sequences. Ann. Missouri Bot. Gard. 82: 176-193.

OlmsteAD, R. G., C. W. DEPAMPHILIS, A. D. WOLFE, N. D. YOUNG, W. J. ELISONS \& P.A. REEVES. 2001. Disintegration of the Scrophulariaceae. Amer. J. Bot. 88: 348-361.

ORTEGA-OLIVENCIA, A. \& J. A. DEVESAALCARAZ. 1992. Estudio palinológico Del gênero Scrophularia L. em La Península Ibérica e Islas Baleares. Acta Bot. Malac. 17: 195-207.

OXELMAN, B., P. KORNNHALL, R. G. OLMSTEAD \& B. BREMER. 2005. Further disintegration of Scrophulariaceae. Taxon 54: 411-425.

POLO, J. M. 1987. Orobanchaceae. In: Valdéz B., Díez M.J., Fernández I. (eds). Atlas Polínico de Andalucia Occidental. Sevilla: Inst. de Desarrollo Regional 


\section{M. Sosa y C. R. Salgado - Palinotaxonomía de Scrophulariaceae sensu lato}

$N^{\circ} 43$ de la Universidad de Sevilla. EXCMA. Diputación de Cádiz. 308-310.

PUNT, W., HOEN, P. P., BLACKMORE, S., NILSSON S. \& A. LE THOMAS. 2007. Glossary of pollen and spore terminology. Rev. Palaeobot. Palynol. 143: $1-81$.

RAHMANZADEH, R. K., K. MULLER, E. FISCHER, D. BARTELS. \& T. BORSCH. 2005. The Linderniaceae and Gratiolaceae are further lineages distinct from the Scrophularaiceae (Lamiales). $P l$. Biol. 7: 67-78.

ROUBIK, D. W. \& J. E. MORENO. 1991. Pollen and Spores of Barro Colorado Island. Monogr. Syst. Bot. Missouri Bot. Gard., St. Louis, Missouri. 36: 268. pp.

SAEIDI-MEHRVAZ, S. \& M. ZARREI. 2006. Pollen morphology of some species of the genus Veronica (Scrophulariaceae) in Irán. Wulfenia 13: 1-9.

SÁENZ LAÍN, C. 2004. Glosario de términos palinológicos. Lazaroa 25: 933-112.

SALGADO, C.R. \& S. M. PIRE. 1997. Morfología polínica de las Buddlejaceae Argentinas. FACENA 13: 59-70.

SALGADO, C. R. \& M. M. SOSA. 2013. Linderniaceae. En Pire, S. M.; Anzótegui, L. M. y A. G. Cuadrado (Eds.) Flora Polínica del Nordeste Argentino. EUDENE. Argentina. ISBN: 978-950-656-149-9, Vol. 4: 111-112.

SANTOS, F. A. R. \& T. S. MELHEM. 1999. Palinologia da Tribo Caprarieae (Scrophulariaceae) do Brasil. Acta Biol. Leopoldensia 21: 175-181.

SANTOS, F. A. R. \& T. S. MELHEM. 2000. Ornamentação do tipo padrão-Croton em grãos de pólen de Scrophulariaceae. Acta Bot. Malac. 25: 81-92.

SCHWARZBACH, A. E. 2004. Plantaginaceae. In "The families and genera of vascular plants”. Ed. K. Kubitzki pp. 327-329- Springer-Berlin.

SOSA, M. M., A. F. PANSERI \& C. R. SALGADO. 2012. Pollen morphology of Stemodia species (Scrophulariaceae) from South America. Palynology 36: $1-9$

SOSA, M. M. \& C. R. SALGADO. 2013a. Orobanchaceae. En Pire, S. M.; Anzótegui, L. M. y A. G. Cuadrado (Eds.) Flora Polínica del Nordeste Argentino. EUDENE. Argentina. ISBN: 978-850-656-149-9, Vol. 4: 113-118.
SOSA, M. M. \& C. R. SALGADO. 2013 b. Scrophulariaceae. En Pire, S. M.; Anzótegui, L. M. y A. G. Cuadrado (Eds.) Flora Polínica del Nordeste Argentino. EUDENE. Argentina. ISBN: 978-850656-149-9, Vol. 4: 137-140.

SOUZA, V. C. \& H. LORENZI. 2008. Botânica Sistemática: guia ilustrado para identifcação das famílias de Fanerógamas nativas e exóticas no Brasil, baseado em APGII. $2^{\text {a }}$. ed. Nova Odessa. Inst. Plantarum.

STEVENS, P. F. 2001 (onwards). Angiosperm Phylogeny Website. Version 12, July 2012-onward. Available at http://www.mobot.org/MOBOT/research/APweb/; 28 July 2014.

TANK, D. C., P. M. BEARDSLEY, S. A. KELCHNER \& R. G. OLMSTEAD. 2006. Review of the systematic of Scrophulariaceae s.l. and their current disposition. Austral. Syst. Bot. 19: 289307.

THIERET, J. W. 1967. Supraspecific Classification in the Scrophulariaceae: a review. SIDA 3(2) 87-106.

VARGHESE, T. M. 1968. Studies in the family Scrophulariaceae- II Pollen morphology. J. Palynol. 4: 91-97.

WANG, H., R. R. MILL \& S. BLACKMORE. 2003. Pollen morphology and infra-generic evolutionary relationships in some Chinese species of Pedicularis (Scrophulariaceae). Pl. Syst. Evol. 237: 1-17.

WANG, H., W. E. YU, J. Q. CHEN \& S. BLACKMORE. 2009. Pollen morphology in relation to floral types and pollination syndromes in Pedicularis (Scrophulariaceae). Pl. Syst. Evol. 277: 153-162.

WETTSTEIN, R. V. 1897. Scrophulariaceae.-In: A. Engler \& K. Prantl (eds.). Die Natürlichen Pflanzenfamilien. IV: 39-107. Engelmann, Leipzig.

ZARE, G., A. A. DÖNMEZ \& E. DÖNMEZ. 2014. Pollen morphology and evolution in the genus Orobanche L. s.l. and its allied genera (Orobancheae/ Orobanchaceae) in Turkey. Pl. Syst. Evol. 300: 783802 .

Recibido el 04 de diciembre de 2015, aceptado el 02 de marzo de 2016. 
